\title{
LA NECESIDAD DE SOPORTE A LA POBLACIÓN DE BARCELONA ANTE EL IMPACTO POTENCIAL DE UN INCREMENTO CLIMÁTICO DE LA TEMPERATU- RA AMBIENTE
}

\author{
José Miguel RASO NADAL \\ Grupo de Climatología. Universitat de Barcelona \\ Juan Carlos PEÑA RABADÁN \\ Servei Meteorològic de Catalunya
}

Recibido: 23/02/2013

Devuelto: $11 / 06 / 2013$

Aceptado: 22/06/2013

RESUMEN: El análisis estadístico de la mortalidad y las temperaturas diarias registradas en Barcelona y su ajuste mediante funciones polinómicas permite establecer la relación existente entre ambas variables y estimar los posibles efectos de un incremento climático de las temperaturas, limitado a uno o dos grados centígrados, que previsiblemente tendrá lugar antes de finalizar el presente siglo, si se cumplen los pronósticos anunciados en los recientes informes del IPCC. Los cálculos realizados ponen de manifiesto la relación entre las temperaturas y el número de fallecimientos y sugieren un futuro incremento de la mortalidad durante el verano y un descenso estimable a lo largo de las restantes estaciones, sobre todo en invierno y primavera. A pesar de que el previsible incremento de las temperaturas pueda acarrear un aumento del número de muertos durante los meses más calurosos del año inferior a los descensos calculados para los restantes meses, resulta totalmente necesario el establecimiento de sistemas eficaces de alerta, aviso y soporte a la población más sensible a las condiciones atmosféricas extremas. Tales sistemas, además de prever e informar ampliamente sobre la inminencia de episodios que comporten riesgos graves para la salud, deben contar con la colaboración de especialistas sanitarios preparados para ayudar a las personas carentes de medios que precisen una especial atención.

PALABRAS CLAVE: Barcelona, cambio climático, mortalidad, ola de calor, riesgos para la salud, sistema de vigilancia y aviso, temperatura.

THE NEED FOR SUPPORT TO THE POPULATION OF BARCELONA FACING THE POTENTIAL IMPACT OF CLIMATE INCREMENT OF AMBIENT TEMPERATURE

ABSTRACT: The statistical analysis of the mortality and the daily temperatures registered in Barcelona and his adjustment by means of polynomial functions allows to establish existing relation between both variables and to estimate the possible effects of a climatic increase of the temperatures, limited to one or two degrees centigrade that, predictably it will take place before finishing the present century, if they are fulfilled the prognoses announced 
in the recent reports of the IPCC. The realized calculations make clear the relation between the temperatures and the number of death and suggest a future increase of the mortality during the summer and an estimable descent along the remaining stations, especially in winter and spring. Although the foreseeable increase of the temperatures could transport an increase of the number of dead persons during the most warm months of the year lower than the descents calculated for the remaining months, there turns out to be completely necessary the establishment of effective systems of alert, notice and support to the population most sensitive to the atmospheric extreme conditions. Such systems must not limit to foreseeing and inform widely about the imminence of episodes that they should endure serious risks for the health, but be provided with the collaboration of sanitary specialists prepared to help the persons lacking in means to need a special attention.

KEY WORDS: Barcelona, climate change, health risk, heatwave, mortality, temperature, watch/warning system.

\section{INTRODUCCIÓN}

El desarrollo óptimo de las funciones orgánicas de los seres humanos, por su condición de homotermos, requiere, entre otros parámetros ambientales, el mantenimiento de la temperatura del aire dentro de unos determinados límites. No obstante, el sistema fisiológico de las personas adultas y sanas dispone de suficientes recursos para hacer frente a fluctuaciones termométricas por encima y debajo de estos límites, aunque sea a costa de padecer, en mayor o menor grado, situaciones de cierto malestar o angustia y, en casos extremos, lesiones de diversa gravedad, incluso irreversibles. Es por esto que las variaciones estacionales y diarias de las temperaturas afectan el estado de bienestar y salud, hasta el punto de, en situaciones extremas, provocar o acelerar procesos patológicos susceptibles de acabar con la vida de algunas personas, especialmente entre las que padecen determinadas enfermedades crónicas, cuentan con una edad avanzada o forman parte de grupos marginales. Estas personas, junto con niños durante su primera infancia, constituyen los denominados grupos de riesgo, por su limitada capacidad de resistencia frente a situaciones atmosféricas extremas. Las personas adultas y sanas prácticamente permanecen al margen de los riesgos de mortalidad relacionadas con las temperaturas del aire en áreas de dominio mediterráneo, como es el caso de Barcelona, pero no es imposible que, insólitamente, alguna de estas personas sea víctima de un golpe de calor, susceptible de provocar su inmediata muerte, una eventualidad que podría aumentar con el cambio climático. Los posibles efectos de los acontecimientos atmosféricos extremos y, en primer lugar, de la temperatura, forman parte de las preocupaciones y conocimientos clásicos de la medicina (BALLESTER, 1996), pero, hasta tiempos bien recientes, los dos o tres últimos decenios, cuando han surgido y proliferado los primeros avisos, seguidos de manifestaciones de alarma, ante los inicios de un cambio climático que la comunidad científica ha acabado por constatar, la atención real a los posibles impactos del clima en la salud hu- 
mana había sido escasa o, a lo sumo, discreta, y estaba lejos de merecer los recientes y actuales esfuerzos dedicados a su estudio. El primero y principal motivo de inquietud se polariza en torno a las temperaturas y efectos de las olas de calor y abundan los estudios en los que se demuestra la existencia de perniciosos efectos de las temperaturas extremas en las personas que constituyen los mencionados grupos de riesgo. A pesar de que la relación entre las temperaturas y la mortalidad analizada en gran número de trabajos referentes a regiones bien diferentes muestren determinados rasgos comunes o semejantes, los efectos del clima y el tiempo atmosférico en la mortalidad difieren sensiblemente de unas comunidades a otras, de manera que los provocados en un determinado país pueden diferir ampliamente de los ocasionados en otro (ANDERSON y BELL, 2009). El alcance de los impactos ocasionados por las temperaturas varía según el nivel educativo, las disponibilidades económicas, el equipamiento de los hogares y la densidad de la población (MEDINA-RAMÓN y SCHWARTZ, 2007; O'NEILl et al., 2003). Algunas de las diferencias entre los efectos en la salud relacionados con las temperaturas no es extraño que resulten paradójicas. Así las temperaturas frías pueden ocasionar menos daños en países del norte de Europa, tradicionalmente preparados para hacer frente a tipos de tiempo fríos, que en regiones mediterráneas con poblaciones normalmente menos equipadas para soportar fuertes descensos termométricos (EUROWINTER GROUP, 1997), y un estudio referido a 11 ciudades de la costa este de los Estados Unidos destaca que los efectos del calor son más apreciables en el nordeste que en el sudeste (CURRIERO et al., 2002). En consecuencia, no cabe esperar que unos efectos de alcance general o que sean aplicables a grandes dominios continentales se cumplan en cada espacio de limitada extensión superficial. De ahí que cualquier aproximación al estudio de los impactos del clima y los avatares atmosféricos en el organismo humano deba realizarse para cada territorio a partir de datos procedentes del mismo, sobre todo si se desea formular una previsión de la posible evolución de tales impactos en el futuro, con el fin de ofrecer a los responsables de la administración pública elementos de juicio ante la necesidad de arbitrar decisiones destinadas a minimizar los posibles efectos negativos sobre la salud. Con este propósito, el presente trabajo se propone un análisis de las relaciones entre las temperaturas y la mortalidad registradas en Barcelona entre finales del siglo pasado y principios del actual destinado a definir una imagen de la situación actual y estimar los posibles efectos sobre la mortalidad en la propia ciudad y territorios más o menos próximos de un incremento climático de las temperaturas, cifrado en uno o dos grados, que, de acuerdo con previsiones realizadas (ALLEN et al., 2009; CALBÓ et al., 2010; MEINSHAUSEN et al., 2009), muy bien podría ocurrir hacia mediados de la presente centuria. 


\section{DATOS Y MÉTODOS}

El interior de la ciudad de Barcelona no dispone ni tan sólo de una estación meteorológica con series termométricas largas y fiables, pero el observatorio de Fabra, situado en un repecho de la montaña del Tibidabo, desde donde se contempla la ciudad a 412 metros sobre el nivel del mar, cuenta con series de temperaturas máximas y mínimas diarias, procedentes de registros seguros realizados desde principios del siglo $\mathrm{XX}$, unos datos que constituyen una imagen válida $\mathrm{o}$, en todo caso difícilmente mejorable, de lo que sucede en el conjunto de la capital. Las series climáticas procedentes de este observatorio, facilitadas por el Servei Meteorològic de Catalunya y la información sobre la mortalidad diaria habida en Barcelona durante el período 1990-2006, proporcionada por la Direcció General de Recursos Sanitaris de la Generalitat de Catalunya han sido utilizadas para poner de manifiesto la posible relación entre las temperaturas y las tasas diarias de defunciones registradas en la ciudad a lo largo de estos 17 años.

Los datos procedentes del observatorio Fabra comprenden las temperaturas máximas y mínimas diarias y la humedad registrada a distintas horas, a partir de los cuales se ha procedido al cálculo de la temperatura media diaria como simple promedio de las temperaturas extremas de cada día y de la denominada temperatura aparente, para cuyo propósito ha sido preciso estimar previamente el punto de rocío, operación realizada a partir de la siguiente expresión:

$$
\operatorname{Pr}=\sqrt[8]{\frac{H}{100}} \cdot(112+0,9 T)+0,1 T-112,
$$

en la que Pr representa el punto de rocío, $T$ la temperatura del aire en grados centígrados y $\mathrm{H}$ la humedad relativa. La temperatura aparente constituye una medida del estado de bienestar o confort que fue desarrollada por STEADMAN (1979) a partir de estudios sobre los efectos de enfriamiento de la piel por evaporación. El cálculo de la temperatura aparente se ha realizado a partir de la siguiente ecuación ya utilizada por KALKSTEIN y VALIMONT en 1986 y más recientemente por diferentes autores (BACCINI et al., 2008; O'NEILL et al., 2005; TAN et al., 2004):

$$
T A=-2,653+0,994 T a+0,0153 T r,
$$

donde $T A$ representa la temperatura aparente, $T a$ es la temperatura del aire y $\operatorname{Tr}$ la temperatura del punto de rocío. 
La información procedente de la Direcció General de Recursos Sanitaris de la Generalitat de Catalunya comprende los registros diarios de defunciones achacables a enfermedades cardiovasculares, respiratorias y a otras causas, anotadas para distintos grupos de edad. Dada la variación de la población de la ciudad a lo largo del período analizado y para facilitar cualquier tipo de comparación con lo sucedido en otros lugares, se ha calculado la proporción de fallecidos por millón de habitantes en relación a la población media de cada año dada por los padrones, censos o estimaciones correspondientes.

Tras constatar, en una primera aproximación, la estacionalidad de la mortalidad, se procederá al ajuste de las series de temperaturas y del número diario de defunciones acaecidas en la ciudad mediante curvas polinómicas con el objetivo de, una vez comprobada su bondad, proceder a la estimación de los posibles efectos de un incremento climático de las temperaturas.

\section{ESTACIONALIDAD DE LA MORTALIDAD}

La representación gráfica de las temperaturas medias diarias calculadas a partir de las observaciones termométricas del período 1990-2006 y de la mortalidad diaria total, la ocurrida entre personas respectivamente mayores y menores de 65 años, así como la atribuible a patologías cardiovasculares, respiratorias y a otras causas por millón de habitantes, tras la suavización de los valores mediante la obtención de las correspondientes medias móviles que comprenden períodos de 15 días y muestra la FIGURA 1, pone claramente de manifiesto que las mayores tasas medias de mortalidad atañen a los primeros y últimos días del año que, por su pertenencia a la estación invernal, son los más fríos. Aunque tanto los episodios extremadamente fríos como cálidos pueden provocar un incremento de la mortalidad, las tasas de defunciones más elevadas, sobre todo entre personas de avanzada edad, suelen registrarse en invierno (KLONER et al., 1999; VAN ROSSUM et al., 2001), y así ocurre en Barcelona, tal como evidencia la aludida figura, en la que se aprecia un acusado descenso de la mortalidad total, así como la habida entre personas mayores de 64 años y la provocada por afecciones cardiovasculares, respiratorias o por otras causas, desde principios del año hasta finales de marzo y un fuerte incremento a partir de la segunda quincena de noviembre que culmina en la postrimerías del año, para enlazar con las elevadas tasas de los primeros días de enero.

La evolución de las curvas representativas de las aludidas tasas de mortalidad total, la registrada entre personas mayores de 64 años y las correspondientes a los tres grupos de causas inmediatas de muerte señaladas, contraria a la descrita por las temperaturas medias, resulta menos acusada entre la se- 
gunda quincena de marzo y principios de junio y, además, muestra algunas irregularidades, la más apreciable de las cuales insinúa un pequeño aumento de los fallecimientos durante los últimos días del propio mes de marzo y los primeros de abril, que acaso no sea ajena a le frecuente inestabilidad atmosférica y la sucesión de episodios termométricos de características contrastadas propia del mes central de la primavera. Entre principios de junio y mediados de septiembre, la evolución de las curvas representativas de las tasas de mortalidad, en contraste con lo que sucede el resto del año, es, en general, similar a la de las temperaturas, con la salvedad del ligero descenso insinuado por las curvas de mortalidad entre finales de junio y primeros días de julio, previo a un ligero ascenso que culmina con la definición de un máximo secundario entre la última década de julio y la primera de agosto, coincidente con el máximo único anual de las temperaturas. Previo a este máximo secundario de mortalidad, se aprecia un mínimo secundario, hacia finales de junio, mientras que el mínimo principal de mortalidad se sitúa en la segunda mitad de septiembre. Las olas de calor y episodios manifiesta o especialmente calurosos no deben ser ajenos al incremento relativo de la mortalidad en Barcelona durante los días más cálidos del año. La incidencia de los episodios excepcionalmente calurosos en la mortalidad ha sido objeto de especial atención en múltiples trabajos a lo largo de los últimos lustros, entre los que caben ser destacados los dedicados a estudiar los impactos en la salud humana de las olas de calor acaecidas en Estados Unidos durante los años 1995 (KARL y KNIGHT, 1997; KELLERMANN y TODD , 1996; KUNKEL et al., 1996; SEMENZA et al., 1996; WHITMAN et al., 1997) y 1999 (KAISER et al., 2001; NAUGHTON et al., 2002; PALECKI et al., 2002). El descenso de la mortalidad en septiembre, al tiempo que las temperaturas inician una evolución decreciente, parece fruto de una concurrencia de dos factores. Por un lado, no suelen suceder en el transcurso del mes episodios termométricos extremos y, por otro, la exacerbación de la mortalidad durante los episodios más calurosos del verano posiblemente provoque un exceso de defunciones entre las personas enfermas más sensibles al calor que, en ausencia de tales episodios, acaecerían pocos días o semanas después y constituirían lo que KALKSTEIN (1993) ha denominado muertes anticipadas, lo cual supondría una merma de óbitos los días posteriores a los más cálidos. Tras el mínimo principal de septiembre $y$, a medida que las temperaturas siguen una clara tendencia descendente, las tasas de mortalidad acusan una evolución ascendente, muy acusada a lo largo del mes de octubre, y un apreciable estancamiento durante la mayor parte de noviembre, hasta que, a finales de este mes, prosiguen el fuerte incremento anteriormente aludido que culmina los días más fríos de diciembre y enero. 
Figura 1. Mortalidad por millón de habitanters y temperatura media diaria calculada a partir de los datos registrados en Barcelona durtante el período 19902006 (Suavización mediante medias móviles centradas cada 15 días)

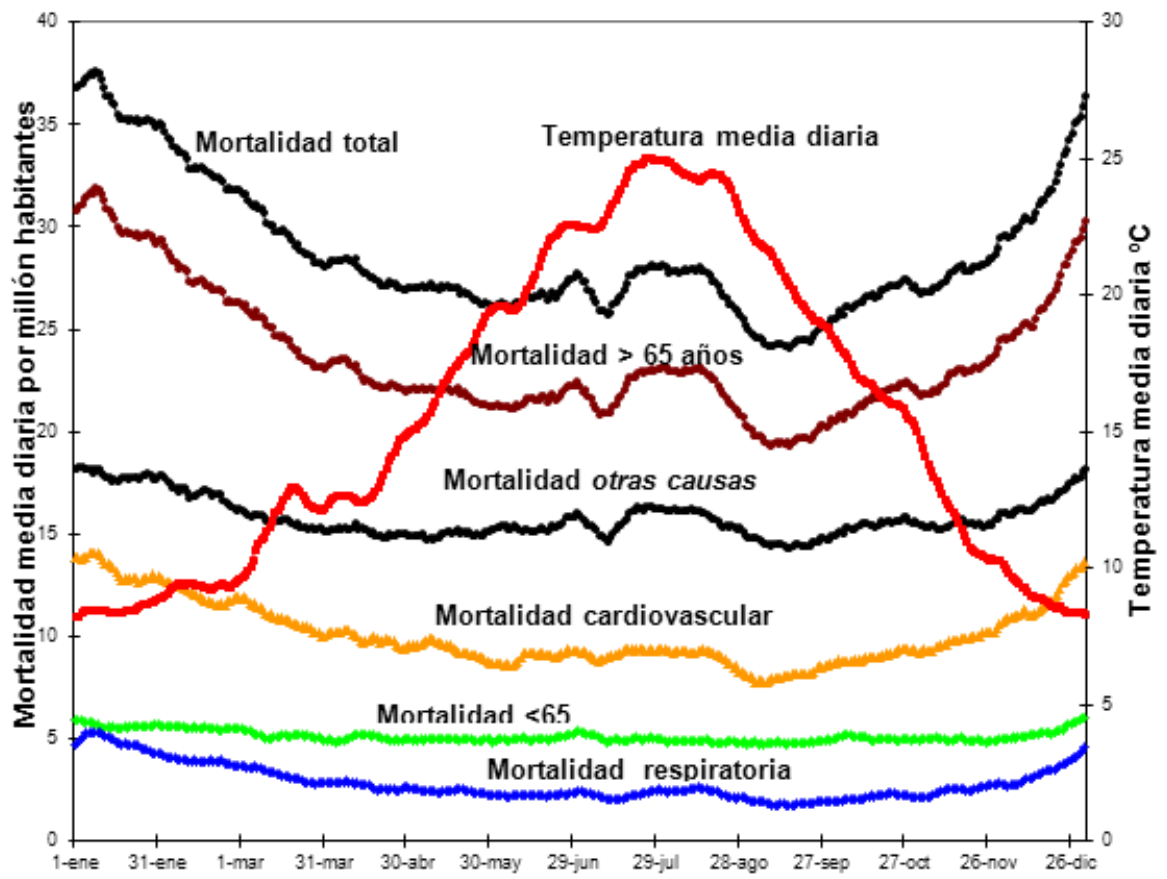

FUENTE: Elaboración propia a partir de series climáticas facilitadas por el Servei Meteorològic de Catalunya y datos sobre mortalidad proporcionados por la Direcció General de Recursos Sanitaris de la Generalitat de Catalunya.

La evolución de la mortalidad por enfermedades respiratorias a lo largo del año no difiere mucho de la total, la cardiovascular o la achacable a otras causas, pero el relativamente reducido número de personas fallecidas por tales padecimientos y una menor sensibilidad de los pacientes víctimas de tales afecciones a los efectos del calor impiden una definición del máximo secundario de verano, seguido del mínimo de septiembre, tan ostensible como el que describen las curvas representativas de estos grupos.

Finalmente, las tasas de mortalidad correspondientes a personas menores de 65 años no sólo difieren, como es natural, en cuantía de las registradas en personas de mayor edad, sino también en su evolución estacional o a lo largo del año, de manera que escasamente apuntan un ligero incremento durante los días más fríos y más cálidos del año. 
La evolución de la mortalidad a lo largo del año evidencia, en definitiva, un ritmo estacional muy marcado, con un ostensible máximo principal, coincidente con los meses más fríos del año, y un discreto, pero claro, máximo secundario, sincrónico con los más cálidos, precedido y seguido por dos mínimos similares, aunque ligeramente más acusado el segundo, localizado el primer mes de otoño. Estos mínimos y máximos, sin embargo, no aparecen definidos en la evolución de la mortalidad entre personas menores de 65 años, que resultan escasamente sensibles a las alternativas termométricas, y tan sólo aparecen ligeramente insinuados en la evolución relativa de las defunciones provocadas por causas respiratorias, que, no obstante, alcanzan un valor máximo anual durante los meses de invierno.

\section{TEMPERATURAS Y TASAS DE MENOR MORTALIDAD}

Aun cuando la correlación lineal entre las temperaturas, tanto aparentes como medias, máximas o mínimas, y el número de fallecimientos habidos durante cada uno de los días del período 1990-2006, correspondientes respectivamente a cada uno de los grupos de edad considerados, se cifre en coeficientes negativos todos ellos y significativos, excepto en el grupo de niños o jóvenes menores de 15 años, cuya inclusión en una amplia tabla parece innecesaria, la estacionalidad de la mortalidad implica que la relación entre las temperaturas y la mortalidad diaria registrada en Barcelona no sea simple, como sucede generalmente en cualesquiera de los territorios situados en latitudes medias. Por este motivo, es frecuente que, en el estudio de las relaciones entre temperaturas y mortalidad, sean muchos los trabajos que aludan a su representación gráfica en forma de una "V" (PAJARES et al., 1997), de una "U” (PAN et al., 1995) o, mejor de una "J" escrita al revés, de manera que la rama de la izquierda sea más larga que la de la derecha (CURRIE y JACUPS, 2003). La parte izquierda de cada curva, descendente, refleja una disminución de la mortalidad a medida que aumentan las temperaturas, mientras que la parte derecha, ascendente, evidencia un progresivo incremento del número de muertos a medida que descienden las temperaturas. Obviamente, la base o parte inferior de este tipo de curvas indica las temperaturas correspondientes a los días que registran unas tasas de mortalidad relativamente más bajas (MCMicHAEL, et al., 2008a). No pocos trabajos realizados para distintos lugares de Europa, América y Australia describen curvas similares (GARCÍA-HERRERA et al., 2005; MEDINA-RAMÓN y SCHWARTZ, 2007; REN et al., 2008; WiLliAMS et al., 2012). Una apariencia en parte semejante a estas curvas es el definido por ajuste polinómico de tercer orden del número diario de muertos por millón de habitantes habido en Barcelona entre 1990 y 2006 y las temperaturas aparentes medias diarias calculadas para el observatorio Fabra (FIGURA 2). 
Figura 2. Relación entre la mortalidad total por millón de habitantes y las temperaturas aparentes medias diarias registradas en Barcelona durante el período 1990-2006. Ajuste polinómico de tercer orden.

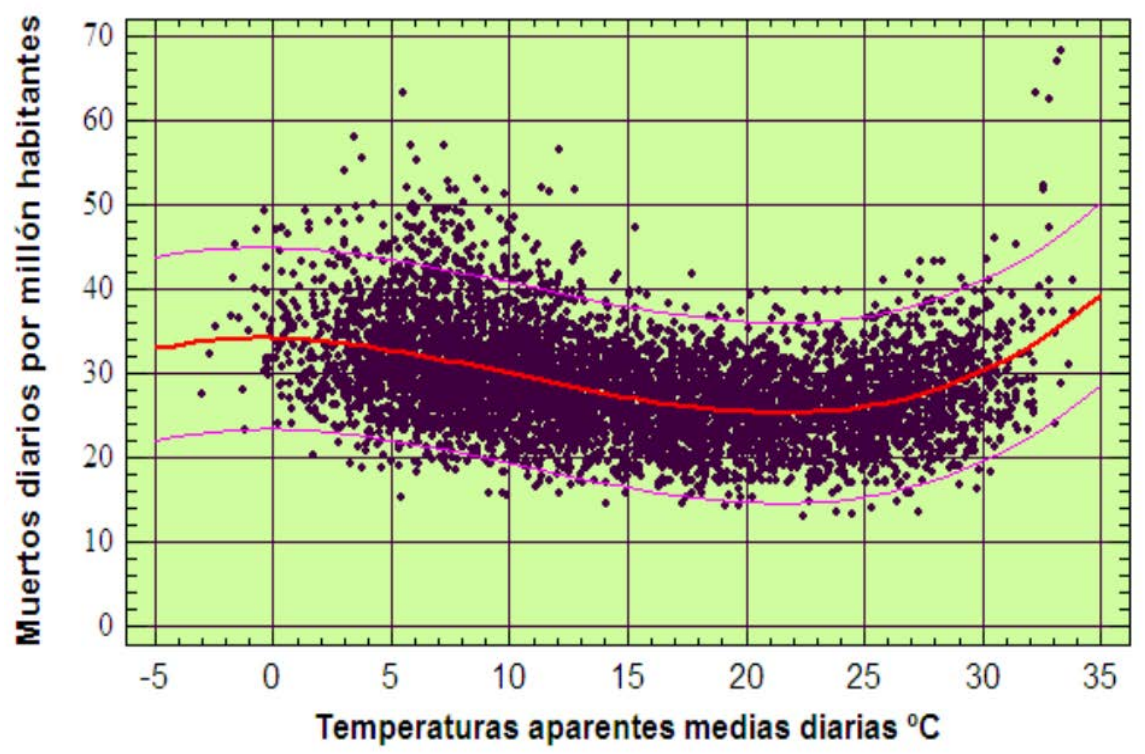

FUENTE: Elaboración propia a partir de series climáticas facilitadas por el Servei Meteorològic de Catalunya y datos sobre mortalidad proporcionados por la Direcció General de Recursos Sanitaris de la Generalitat de Catalunya.

La curva polinómica muestra dos inflexiones, una principal, correspondiente con la única de las representaciones gráficas aludidas en los trabajos precedentes, que indica las temperaturas bajo las cuales se registran las menores tasas medias de mortalidad, y una secundaria correspondiente a los días con temperaturas ostensiblemente bajas. La principal o más acusada inflexión de la curva polinómica pone de manifiesto que la menor cuantía de las tasas medias de mortalidad corresponde a días con unas temperaturas aparentes en torno a los $20-21^{\circ} \mathrm{C}$. Como resulta evidente, todo alejamiento de las temperaturas de este umbral, va acompañado de un incremento general de las tasas de mortalidad, pero, mientras los ascensos termométricos van acompañados de un manifiesto crecimiento de las tasas de mortalidad, las temperaturas de inferior cuantía únicamente muestran una relación claramente negativa con la mortalidad entre los aproximadamente $3^{\circ}$ y $20^{\circ} \mathrm{C}$ de temperatura aparente, de manera que el descenso de esta comporta una disminución de la mortalidad media. La segunda inflexión de la curva polinómica se sitúa en torno a los $0^{\circ} \mathrm{C}$, temperaturas aparentes bajo las cuales se aprecian unas tasas de mortalidad similar o escasamente inferiores a las más 
elevadas registradas durante el período analizado. Por consiguiente, aun cuando haya sido destacado en multitud de estudios la existencia de determinadas temperaturas o intervalos termométricos propios de cada territorio, que en el caso de Barcelona puede cifrarse entre 20 y $21^{\circ} \mathrm{C}$, bajo cuyo predominio, las tasas medias de mortalidad registran unos valores medios relativamente inferiores a los padecidos cuando las temperaturas se sitúan tanto por debajo como por encima de tales intervalos y que, generalmente se puede apreciar un incremento de la mortalidad a medida que se alejan de los mismos, el ascenso del número de fallecidos con el descenso de la temperaturas resulta menos evidente, precisamente los días que merecen ser calificados como muy o excepcionalmente fríos. Aunque el incremento de los efectos negativos en la salud con el descenso de las temperaturas es indudable, y así ha sido constatado en la gran mayoría o casi totalidad de los trabajos existentes que estudian la relación entre ambas variables, también resulta posible una atenuación o, al menos, una moderación del acrecentamiento de la mortalidad precisamente cuando las temperaturas se sitúan por debajo de determinados umbrales. La atenuación del incremento de los efectos negativos de las temperaturas extremadamente bajas sobre la salud ya fue observada en un estudio acerca del número de emergencias hospitalarias en la ciudad japonesa de Fukuoka, en el que sus autores constataron un aumento drástico en el número de admisiones urgentes en centros clínicos con el descenso de las temperaturas por debajo de $12^{\circ} \mathrm{C}$, hasta alcanzar una meseta a $4^{\circ} \mathrm{C}$ (MAKIE et al., 2002). La merma en el incremento de la mortalidad en Barcelona cuando las temperaturas son especial o extraordinariamente frías, o del número de emergencias en Fukuoka cuando no alcanzan los $4^{\circ} \mathrm{C}$, no debe interpretarse como que los impactos negativos de los descensos termométricos por debajo de determinados umbrales dejen de constituir unos riesgos potencialmente crecientes para la salud con sucesivas disminuciones de la temperaturas. Posiblemente dos circunstancias o fenómenos condicionan los resultados de los cálculos que enmascaran este riesgo. En primer lugar, las temperaturas extremadamente frías suelen registrarse tras algunas jornadas menos frías pero susceptibles de provocar las denominadas por KALKSTEIN (1993) muertes anticipadas, esencialmente acaecidas entre ancianos, enfermos crónicos, grupos marginales y, en sociedades carentes de medios, niños en su primera infancia, es decir entre las personas más sensibles a los impactos de las temperaturas extremas. Se trata de muertes que, bajo condiciones atmosféricas favorables, probablemente ocurrirían en un breve espacio de tiempo o escasamente pocos días después de su acaecimiento real. Además, los impactos de las temperaturas extremadamente bajas susceptibles de alterar la salud de las personas hasta acabar con su vida, no provocan este resultado de manera inmediata, sino con un mayor o menor retraso de compleja evaluación. En segundo término, la previsión o inminencia de las olas de frío o fuertes des- 
censos termométricos motiva la adopción de medidas preventivas entre las personas más sensibles a los avatares termométricos, muchas de las cuales procuran protegerse, en mayor o menor medida, de las temperaturas extremadamente bajas cuando advierten o son conscientes de su peligro. Finalmente, las mayores tasas de mortalidad que se observan durante los meses de invierno parecen responder en no menor medida a la continuidad de las temperaturas relativamente bajas, que al advenimiento de episodios extremadamente fríos.

El CUADRO 1 muestra las ecuaciones de ajuste polinómico de tercer orden calculadas para distintas tasas de mortalidad diaria por millón de habitantes en relación, tanto con las temperaturas aparentes, como con las medias diarias. Las tasas de mortalidad corresponden respectivamente al número total de fallecimientos por millón de habitantes ocurridos cada día (mortalidad total), las muertes provocadas por enfermedades o fallos orgánicos de origen cardiovascular (mortalidad cardiovascular), las ocasionadas por padecimientos respiratorios (mortalidad respiratoria) y, finalmente, las sobrevenidas por cualesquiera otras causas. A partir de estas ecuaciones es posible estimar como temperaturas óptimas para la salud las situadas en torno a los $20,5^{\circ} \mathrm{C}$, por cuanto que bajo las mismas se registran en general las menores tasas de mortalidad, tanto en el caso del ajuste calculado con relación a las temperaturas aparentes como con las medias diarias registradas en el observatorio de Fabra. Por encima de este umbral, a todo incremento de las temperaturas, aparentes o medias, corresponde un aumento de las tasas de mortalidad. En cambio, por debajo de los $20,5^{\circ} \mathrm{C}$, existe una relación general de signo negativo entre las tasas de mortalidad y las temperaturas que cesa cuando las temperaturas aparentes no alcanzan $-1,0^{\circ} \mathrm{C}$, tal como sugiere el gráfico de la FIGURA 2 y ha sido destacado anteriormente, o cuando las medias diarias no sobrepasan los $5,0^{\circ} \mathrm{C}$. La tabla indica además el valor $p$ en el análisis de variancia (ANOVA) y el porcentaje de variancia explicada, muestra la bondad del ajuste y evidencia la mayor relación de las temperaturas con la mortalidad provocada por fallos cardiovasculares o respiratorios que la habida bajo otras causas, estimada en valores insignificantes, de manera que los impactos de las temperaturas escasamente muestran relación con las tasas de mortalidad debida a causas distintas a las de origen cardiovascular o respiratorio. Finalmente, merece ser destacado el contraste entre la variancia explicada en el ajuste de la mortalidad entre personas mayores y menores de 65 años, del cual puede deducirse que las temperaturas en general, incluso las relativa 0 excepcionalmente altas o bajas, no representan un riesgo apreciable entre las personas que no alcanzan esta edad, al menos en cuanto al acortamiento de su vida. 
Cuadro 1. Ecuaciones de ajuste polinómico de tercer orden de la mortalidad por millón de habitantes y las temperaturas diarias registradas en Barcelona durante el período 1990-2006. (MT=mortalidad total; MC=idem provocada por causas cardiovasculares; $M R=i d e m$ provocada por causas respiratorias; $M X=$ idem provocada por "otras causas"; $T A=$ temperatura aparente; TM=temperatura media; $T X=$ temperatura máxima; TI=temperatura mínima.

\begin{tabular}{|c|c|c|}
\hline $\begin{array}{l}\text { Ecuaciones de ajuste calculadas para la mortalidad total por millón } \\
\text { de habitantes }\end{array}$ & $A$ & B \\
\hline $\mathrm{MT}=34,1693-0,0574552 \mathrm{TA}-0,0519523 \mathrm{TA}^{2}+0,00165322 \mathrm{TA}^{3}$ & 0,0000 & 19,44 \\
\hline $\mathrm{MC}=12,7748-0,0288795 \mathrm{TA}-0,0232458 \mathrm{TA} \mathrm{A}^{2}+0,000706495 \mathrm{TA}^{3}$ & 0,0000 & 16,95 \\
\hline $\mathrm{MR}=4,09955-0,0104436 \mathrm{TA}-0,0117849 \mathrm{TA} \mathrm{A}^{2}+0,000360123 \mathrm{TA} \mathrm{A}^{3}$ & 0,0000 & 12,78 \\
\hline $\mathrm{MX}=17,3001-0,0190518 \mathrm{TA}-0,0168353 \mathrm{TA} \mathrm{A}^{2}+0,000584473 \mathrm{TA} \mathrm{A}^{3}$ & 0,0000 & 4,87 \\
\hline $\mathrm{MT}=32,7226+0,772932 \mathrm{TM}-0,125085 \mathrm{TM}^{2}+0,00342534 \mathrm{TM}^{3}$ & 0,0000 & 19,84 \\
\hline $\mathrm{MC}=12,101+0,340482 * \mathrm{TM}-0,0546311 \mathrm{TM}^{2}+0,00144018 \mathrm{TM}^{3}$ & 0,0000 & 15,92 \\
\hline $\mathrm{MR}=3,88816+0,144216 \mathrm{TM}-0,0256121 \mathrm{TM}^{2}+0,000694641 \mathrm{TM}^{3}$ & 0,0000 & 12,61 \\
\hline $\mathrm{MX}=16,7367+0,287685 \mathrm{TM}-0,0447758 \mathrm{TM}^{2}+0,0012886 \mathrm{TM}^{3}$ & 0,0000 & 5,62 \\
\hline $\begin{array}{l}\text { Ecuaciones de ajuste calculadas para la mortalidad total por millón } \\
\text { de habitantes entre personas mayores de } 64 \text { años }\end{array}$ & A & $B$ \\
\hline MT $>65=28,5251-0,0218632 *$ TA-0,0509538TA ${ }^{2}+0,00160669 \mathrm{TA}^{3}$ & 0,0000 & 19,18 \\
\hline $\begin{array}{l}\text { Ecuaciones de ajuste calculadas para la mortalidad total por millón } \\
\text { de habitantes entre personas menores de } 65 \text { años }\end{array}$ & A & B \\
\hline $\mathrm{MT}<65=5,64132-0,0350984 \mathrm{TA}-0,0010328 \mathrm{TA}^{2}+0,000047306 \mathrm{TA}^{3}$ & 0,0000 & 1,17 \\
\hline , Valor $p$ en el ANOVA; B, Explicación Variancia (\%) & & \\
\hline
\end{tabular}

En resumen, aunque las temperaturas, incluso las extremadamente altas o bajas, raramente son capaces de provocar la muerte de personas sanas, es evidente que pueden interferir el estado de salud de individuos víctimas de algún tipo de padecimientos cardiovasculares o respiratorios, particularmente entre mayores de 64 años.

\section{IMPACTO DE LAS TEMPERATURAS RELATIVA Y EXTRE- MADAMENTE ALTAS: LOS EPISODIOS Y OLAS DE CALOR}

Los efectos sobre la salud, y particularmente sobre la mortalidad, de los episodios excepcionalmente cálidos son inmediatos o se manifiestan dentro de un reducido número de días posteriores a los eventos de excepcional calor (HALES et al., 2003). Los más graves y evidentes de los efectos de estos episodios son los denominados golpes de calor, al riesgo de los cuales están sometidas todas las personas, sobre todo si realizan actividades físicas de cierta intensidad al aire libre mientras los termómetros alcanzan valores manifiestamente elevados, circunstancia bajo la que es posible el advenimiento de la muerte en un corto espacio de tiempo. El número de defunciones provocado por golpes de calor es reducido y no suele existir constancia de su ocurrencia en las tasas de mortalidad disponibles. En cambio, no son raras 
las muertes acaecidas como consecuencia de trastornos cardiovasculares o respiratorios desencadenados o agravados por efectos de temperaturas extremadamente altas. En todo caso, los incrementos de la mortalidad relacionados con el calor se manifiestan mientras las temperaturas mantienen unos valores ostensiblemente superiores a los medios de los días más cálidos del año o dentro de los días inmediatos posteriores. Además, los efectos de las temperaturas en el organismo humano pueden resultar enmascarados e incluso alterados por efecto de la contaminación y ha sido reconocido un ligero efecto de confusión provocado por el ozono y las partículas presentes en el aire respirado (O’NeILl et al. 2005). La presencia de ozono en la baja troposfera, cuyos efectos negativos sobre la salud humana, particularmente por su impacto en el aparato respiratorio, son indudables, no es ajena a la existencia de elevadas temperaturas que facilitan su síntesis a partir de determinados precursores, particularmente en ambientes urbanos donde no faltan óxidos de nitrógeno resultantes de la combustión de productos fósiles. Otros autores han detectado un moderado grado de confusión provocado por el ozono, pero ningún efecto modificador atribuible a este gas o a las partículas (BASU et al., 2008; ZANOBETTI y SCHWARTZ, 2008), o bien reconocen una significativa modificación provocada únicamente por las partículas en determinados enfermos (REN et al., 2011). Cualesquiera que sean los efectos específicos de las temperaturas altas sobre la salud, ya sean los esencial o exclusivamente provocados por el calor del cual son reflejo o fruto, en mayor o menor medida, de la acción de determinados contaminantes, cuya presencia en la baja atmósfera está condicionada por las propias temperaturas, los efectos de los ascensos termométricos más o menos acusados sobre la mortalidad son indudables y se polarizan en las personas de edad avanzada.

No es fácil determinar en qué medida contribuyen las temperaturas extraordinariamente altas al desencadenamiento de procesos patológicos con resultado de muerte. Más dificultades conllevaría todo intento de evaluar con precisión el papel correspondiente al calor en la generación de estados de fatiga y angustia, así como la subsiguiente pérdida de reacción, responsables de no pocos accidentes graves los días más opresivos del verano. La diversidad de causas de muerte relacionadas con el calor y la difícil, rara o inexistente identificación de la intervención del calor en el registro de las causas de las defunciones imposibilitan determinar el número preciso de víctimas en cada episodio de calor, razón por la cual suelen estimarse como tales, las integrantes del denominado exceso de mortalidad, equivalente a la diferencia entre las muertes esperadas o medias y las registradas los días en que las temperaturas mantienen unos valores excepcionalmente altos. Multitud de trabajos referentes a diferentes países y ciudades de Europa, América y Australia han destacado que las temperaturas más altas del verano pueden pro- 
vocar un inmediato incremento de la mortalidad (BASU et al., 2008; BELL et al., 2008; DESSAI, 2002; MCMICHAEL et al., 2008b; MEDINA-RAMÓN y SCHWARTZ, 2007; Michelozzi et al., 2010; PATTENDEN et al., 2003; STAFOGGIA et al., 2006; VANECKOVA et al., 2008). Ese incremento coincide en el tiempo con los días más calurosos, especialmente si forman parte de una ola de calor, o durante los inmediatos siguientes a tal evento. En general, se entiende como ola de calor todo episodio de intenso y súbito incremento termométrico, provocado por la invasión de una masa de aire caliente que afecta áreas relativamente amplias y suele remitir entre unos pocos y no más de unos diez o quince días. Aunque no existe una universal o amplia aceptación en cuanto a los umbrales que han de sobrepasar las temperaturas, ni en cuanto a la duración de tales episodios para que merezcan ser reconocidos como constituyentes de una ola de calor, los límites suelen fijarse generalmente con relación a un determinado percentil no inferior al nonagésimo quinto o fijados en una cuantía que supere a la media de cada día en, al menos, cinco o seis grados. Esto significa que su valor depende del clima de cada lugar, circunstancia que es imprescindible tener en cuenta, ya que temperaturas potencialmente agresivas para la salud en un país frío de latitudes altas pueden resultar confortables en regiones cálidas de latitudes bajas. Así, mientras los meteorólogos canadienses consideran, en sus previsiones, como olas de calor los episodios de tres o más días con temperaturas máximas superiores a $32^{\circ} \mathrm{C}$, los holandeses aceptan como tales toda sucesión de al menos cinco días consecutivos con temperaturas máximas superiores a $25^{\circ} \mathrm{C}$, siempre que tres o más sobrepasen los $32^{\circ} \mathrm{C}$, y los responsables médicos de Dallas definen un ola de calor como la persistencia de temperaturas máximas por encima de $37,8^{\circ} \mathrm{C}$ tres o más días consecutivos (HALES et al., 2003). Definiciones bajo criterios similares son válidas para diferentes áreas, pero no intercambiables por cifrarse en valores de desigual significación en regiones de climas diferentes. Efectivamente, los umbrales termométricos por encima de los cuales puede apreciarse un destacable incremento de la mortalidad relacionado con el calor varían considerablemente de unas ciudades a otras (CURRIERo et al., 2002; SEMENZA et al., 1999). En los Estados Unidos, este umbral ha sido estimado en $32^{\circ} \mathrm{C}$ para la ciudad septentrional de Detroit y en $36^{\circ} \mathrm{C}$ para la meridional de Saint Louis (KALKSTEIN y VALIMONT, 1986). Estudios realizados para diferentes ciudades de la península Ibérica han reconocido incrementos apreciables del número de óbitos a partir de ascensos de las temperaturas que para distintos puntos de España oscilan entre los $26,2^{\circ} \mathrm{C}$ de La Coruña y los $41,2^{\circ} \mathrm{C}$ de Córdoba (DÍAZ et al., 2005), mientras que en Lisboa se cifran en $33,5^{\circ} \mathrm{C}$ (GARCÍA-HERRERA et al., 2005). En Barcelona, estos ascensos pueden estimarse en torno o por encima de los $33,0^{\circ} \mathrm{C}$ (PEÑA y RASO, 2008). A falta de una definición universal de ola de calor, en el estudio de los efectos de las temperaturas extremadamente altas en la sa- 
lud humana, puede catalogarse como ola de calor todo episodio atmosférico caracterizado por unas temperaturas suficientemente altas como para provocar o contribuir a un incremento del número de defunciones o de emergencias hospitalarias. Así lo entendieron los autores de los trabajos que se publicaron sobre las olas de calor extremo que padecieron amplias áreas de Estados Unidos el mes de julio de 1995 y sus impactos en la mortalidad (CHANGNON et al., 1996; KARL y KNIGHT, 1997; KUNKEL et al., 1996; SEMENZA, 1996; SEMENZA et al., 1996; WHITMAN et al., 1997). Una de las ciudades más castigadas fue Chicago, donde las muertes atribuidas al calor se cifraron entre 500 y 800, según los trabajos consultados, y las emergencias hospitalarias computaron 3.300 ingresos. En el mismo año 1995, Inglaterra también padeció los efectos del calor y Londres registró un incremento de la mortalidad del 16,1 \% durante las olas de calor que tuvieron lugar los meses de julio y agosto (RoONEY et al., 1998). Años antes, en 1987, una ola de calor castigó Atenas con un incremento de la mortalidad por causa del calor evaluado en 2000 personas (KATSOUYANNI et al., 1988) y, en 1994, una fuerte ola de calor provocó en Bélgica un acusado incremento de las muertes entre personas de edad avanzada (SARTOR et al., 1995). La India padece las consecuencias de los episodios de calor todos los años. A uno de los más severos de estos eventos se atribuyó la provocación de 2600 muertes, número que, según fuentes no oficiales, fue de casi el doble (KUMAR, 1998).

Excepcionalmente drásticos fueron los efectos de las severas olas de calor que padecieron la mayoría de los países de Europa occidental y meridional durante el verano de 2003, con temperaturas máximas próximas e incluso superiores a $40^{\circ} \mathrm{C}$ en repetidas jornadas, especialmente los meses de junio y agosto. Los territorios situados entre el norte de las penínsulas Ibérica e Itálica y el centro del continente fueron los más castigados y tuvieron que soportar episodios de calor extremo durante toda la estación, pero fue justamente el mes de agosto de 2003, cuando casi todo el continente europeo padeció una ola de calor sin precedentes, registrándose el mayor impacto sobre la mortalidad nunca conocido hasta aquel año (SCHÄR y JENDRITZKY, 2004). La incertidumbre de los incrementos de la mortalidad atribuibles a las temperaturas extremas y la falta de datos fiables, ocasionalmente por razones políticas, subestiman estos efectos y ofrecen valoraciones discrepantes. Es por este motivo que, según las fuentes consultadas, la metodología adoptada y el período de referencia, las estimaciones del número de muertos provocados por las olas de calor del año 2003 en Europa difieren ostensiblemente. Así, mientras KOVATS y JENDRITZKY (2006) consideran que este número osciló entre 27.000 y 40.000, RoBINE et al. (2008), haciéndose eco de informaciones procedentes de diferentes institutos estadísticos publicadas en la prensa, sitúan el exceso de la mortalidad virtualmente imputable al calor en 
más de 70.000 defunciones, de las cuales, 20.089 se registraron en Italia, 19.490 en Francia, 15.090 en España y 9.355 en Alemania. Sin embargo, MARTíNEZ-NAVARRO et al. (2004), a partir de los registros procedentes de todas las capitales de provincia de España y una muestra aleatoria de 107 municipios, estimaron los excesos de mortalidad en 3.166 personas, cantidad que representa un incremento del 7,9 \% respecto a la mortalidad esperada, mientras asignaban a Barcelona un exceso de 665 muertes, un total superior en un 16,6 \% al número de muertes esperadas. Un trabajo posterior reduce el número de muertos por efectos de las olas de calor del año 2003 en Barcelona, al tener en cuenta la acción de los contaminantes fotoquímicos, pero mantiene la estimación de las defunciones provocadas por el calor excepcional de este año por encima de 500 (ToBIAS et al., 2010), una cantidad suficientemente importante para que, junto con los porcentajes aludidos, claramente superiores a los del conjunto de España, constituya una evidencia manifiesta de la intensidad de los citados episodios de calor extremo en Cataluña. Por otro lado, una de las razones señaladas como explicación, al menos en cierta medida, del elevado número de fallecimientos ocurridos en Barcelona durante el verano de 2003, fue la falta de gripe durante el invierno precedente (SUNYER, 2008), ya que una baja mortalidad de invierno, al mermar el número de muertes entre las personas incluidas en los consabidos grupos de riesgo, particularmente las de mayor edad, las más susceptibles al riesgo de morir bajo los efectos de las temperaturas extremas, permite que un mayor número de estas personas estén sometidas a las altas termométricas del verano siguiente (RoCKLÖV et al., 2009).

\section{IMPACTOS POTENCIALES PARA LA SALUD DE UN MODERADO INCREMENTO DE LAS TEMPRATURAS}

Sabido es el amplio consenso existente acerca del actual y probable futuro calentamiento del planeta que, a finales del presente siglo, podría cifrarse en un incremento de las temperaturas medias próximo a los $6^{\circ} \mathrm{C}$, en el supuesto de cumplirse las previsiones más pesimistas expuestas en el último informe del IPCC (2007). Dado el indudable efecto que las temperaturas ejercen sobre la salud humana hasta el punto de exacerbar o, acaso, provocar los procesos trastornos patológicos con final irreversible, resulta conveniente y necesario prever la posibles consecuencias que acarreará el progresivo calentamiento global en cada territorio, de acuerdo, en primer lugar, con el posible alcance del cambio climático previsible y, en segundo término, con las condiciones sociales y económicas de las sociedades que acogen.

A fin de proceder a una estimación de los posibles impactos del calentamiento del clima en la salud de los habitantes de Barcelona, se ha planteado una valoración de las consecuencias para la mortalidad de incrementos ter- 
mométricos limitados a uno o dos grados que, en caso de llegar a producirse las peores previsiones anunciadas en el aludido informa del IPCC, serán ampliamente rebasados a finales de la actual centuria, mientras que proyecciones recientes sugieren que aumentos de este orden se alcancen en el litoral mediterráneo de la Península Ibérica hacia mediados del presente siglo (BRUNET et al., 2009; CALBó et al., 2010).

Cuadro 2. Variación estimada por ajuste polinómico de tercer orden de la mortalidad total y estacional por millón de habitantes en Barcelona, según causas.

A. Supuesto de un incremento de las temperaturas aparentes y medias diarias en un grado

\begin{tabular}{lrrrrrrrr}
\hline & \multicolumn{3}{c}{ Ajuste con temperaturas aparentes } & \multicolumn{4}{c}{ Ajuste con temperaturas medias } \\
\cline { 2 - 9 } & \multicolumn{1}{c}{$M T$} & \multicolumn{1}{c}{$M C$} & \multicolumn{1}{c}{$M R$} & \multicolumn{1}{c}{$M X$} & \multicolumn{1}{l}{$M T$} & \multicolumn{1}{c}{$M C$} & \multicolumn{1}{c}{$M R$} & \multicolumn{1}{c}{$M X$} \\
\hline Total anual & $-81,0$ & $-48,0$ & $-22,2$ & $-10,9$ & $-96,5$ & $-59,1$ & $-27,1$ & $-10,7$ \\
\hline Invierno & $-49,5$ & $-22,9$ & $-11,2$ & $-15,4$ & $-60,7$ & $-27,6$ & $-13,9$ & $-19,2$ \\
\hline Verano & 43,6 & 13,5 & 7,5 & 22,5 & 56,2 & 16,2 & 9,2 & 30,7 \\
\hline Primavera & $-47,5$ & $-23,2$ & $-11,2$ & $-13,0$ & $-56,8$ & $-27,9$ & $-13,3$ & $-15,5$ \\
\hline Otoño & $-27,6$ & $-15,2$ & $-7,2$ & $-5,1$ & $-35,5$ & $-19,7$ & $-9,1$ & $-6-7$ \\
\hline
\end{tabular}

B. Supuesto de un incremento de las temperaturas aparentes y medias diarias en dos grados

\begin{tabular}{lrrrrrrrr}
\hline & \multicolumn{3}{c}{ Ajuste con temperaturas aparentes } & \multicolumn{4}{c}{ Ajuste con temperaturas medias } \\
\cline { 2 - 9 } & \multicolumn{1}{c}{$M T$} & \multicolumn{1}{c}{$M C$} & \multicolumn{1}{c}{$M R$} & \multicolumn{1}{c}{$M X$} & \multicolumn{1}{c}{$M T$} & \multicolumn{1}{c}{$M C$} & \multicolumn{1}{c}{$M R$} & \multicolumn{1}{c}{$M X$} \\
\hline Total anual & $-142,2$ & $-88,3$ & $-40,8$ & $-13,6$ & $-160,6$ & $-105,6$ & $-47,7$ & $-7,1$ \\
\hline Invierno & $-101,5$ & $-47,1$ & $-23,0$ & $-31,4$ & $-125,6$ & $-57,4$ & $-28,6$ & $-39,6$ \\
\hline Verano & 101,0 & 32,6 & 18,0 & 50,3 & 134,8 & 41,5 & 22,9 & 70,3 \\
\hline Primavera & $-92,3$ & $-45,4$ & $-22,1$ & $-24,7$ & $-108,1$ & $-53,9$ & $-25,6$ & $-28,6$ \\
\hline Otoño & $-49,3$ & $-28,3$ & $-13,3$ & $-7,8$ & $-61,4$ & $-35,8$ & $-16,3$ & $-9,3$ \\
\hline
\end{tabular}

(MT: mortalidad total por millón de habitantes; MC: ídem provocada por causas cardiovasculares; MR: ídem provocada por causas respiratorias; MX: ídem provocada por "otras causas")

FUENTE: Elaboración propia a partir de series climáticas facilitadas por el Servei Meteorològic de Catalunya y datos sobre mortalidad proporcionados por la Direcció General de Recursos Sanitaris de la Generalitat de Catalunya

La estimación de las posibles consecuencias de incrementos termométricos dentro de los umbrales señalados ha sido obtenida a partir de los ajustes polinómicos de tercer orden indicados en el anteriormente aludido CUADRO 1. Los cálculos han sido realizados con datos de temperatura aparente y media diaria correspondientes al conjunto del año y a cada una de las estaciones, en relación tanto a la mortalidad sobrevenida por fallos de origen cardiovascular o respiratorio, como a la debida a otras causas. Los resultados así obtenidos que muestra la TABLA 2 indican que el aumento de un grado de las temperaturas diarias, medias o aparentes, puede suponer una reducción de las tasas anuales de mortalidad por millón de habitantes del orden de unas 80 a 100 personas. Un 60 \% de la reducción total de la mortalidad sería fruto de una importante disminución de fallecimientos por afecciones cardiovasculares y casi un $30 \%$ por un descenso de los achacables a patologías respirato- 
rias, mientras que los óbitos debidos a otras causas tan sólo supondrían una contracción de un 10 \% aproximadamente. La reducción de la mortalidad tendría lugar principalmente en invierno y en primavera, proporciones próximas o, en general, escasamente inferiores al $40 \%$ en cada una de las estaciones, tanto en la provocada por afecciones cardiovasculares como respiratorias o la ocasionada por otras causas, mientras que en otoño la disminución de los fallecimientos se situaría en proporciones próximas o escasamente superiores al 20\%. En cambio, durante el verano, probablemente se produciría un incremento del número de fallecimientos de en torno a 50 personas, según las estimaciones realizadas por ajuste con las temperaturas aparentes o medias diarias respectivamente. El incremento de la mortalidad en verano por causas cardiovasculares representaría sobre un $30 \%$ del aumento, mientras que las afecciones respiratorias serían responsables de aproximadamente entre un 15 y un $20 \%$ del aumento, mientras que poco más de la mitad del mismo sería el relacionado con otras causas.

De un mayor incremento de las temperaturas, naturalmente cabría esperar unos efectos más acusados en la mortalidad. Así, en el supuesto de que el aumento alcanzara una media de dos grados, las estimaciones realizadas por ajuste con curvas polinómicas de tercer orden indican que los impactos en el número de fallecimientos podrían concretarse en una reducción de la mortalidad próxima a las 150 personas por millón de habitantes. La disminución posiblemente tendría lugar fundamentalmente durante el invierno y la primavera, de manera que podría suponer una merma de en torno a un centenar de muertes por millón en invierno y una cantidad similar o tan sólo ligeramente inferior en primavera, mientras que el descenso se reduciría en otoño a valores equivalentes a aproximadamente la mitad de los ocurridos en las dos estaciones citadas. A la disminución de la mortalidad contribuiría, en primer lugar, la proporción de los fallecidos por causas cardiovasculares, cuya reducción supondría aproximadamente el $60 \%$ del total, en tanto que entre los fallecimientos por causas respiratorias podrían suponer un $30 \%$ y, en torno al $10 \%$ restante, los debidos a otras causas. Dentro de las tres estaciones que se beneficiarían de un descenso de la mortalidad, las contracciones más importantes corresponderían a los susceptibles de morir por causas cardiovasculares que representarían poco menos o alrededor de la mitad de las estimadas para cada una de las estaciones. La reducción de los fallecimientos de origen respiratorio y los provocados por otras causas alcanzaría entre el 20 y el $30 \%$ de las muertes evitadas durante las tres estaciones y porcentajes de orden parecido supondrían las relacionadas con otras causas, excepto en otoño, cuando únicamente representarían sobre un $15 \%$. En cambio, el incremento de dos grados en las temperaturas comportaría un claro incremento de la mortalidad en verano, que podría superar el centenar de personas por 
millón de habitantes, de las cuales, un tercio serían víctimas de afecciones cardiovasculares, poco menos del $20 \%$ de patología respiratorias y alrededor de la mitad del incremento de fallecimientos sería ocasionados por otras causas.

Cuadro 3. Variación estimada por ajuste polinómico de tercer orden de la mortalidad por millón de habitantes en Barcelona para distintos grupos de edad.

\begin{tabular}{lcccccccc}
\hline & A. Supuesto de un incremento de las temperaturas aparentes y medias diarias en un grado \\
\cline { 2 - 10 } & \multicolumn{4}{c}{ Ajuste con temperaturas aparentes } & \multicolumn{4}{c}{ Ajuste con temperaturas medias } \\
\cline { 2 - 10 } & $<65$ & $65-74$ & $75-84$ & $>84$ & $<65$ & $65-74$ & $75-84$ & $>84$ \\
& años & \multicolumn{1}{c}{ años } & \multicolumn{1}{c}{ años } & años & \multicolumn{1}{c}{ años } & \multicolumn{1}{c}{ años } & \multicolumn{1}{c}{ años } & años \\
\hline Total anual & $-9,4$ & $-16,5$ & $-27,0$ & $-28,0$ & $-11,5$ & $-20,4$ & $-34,4$ & $-32,3$ \\
\hline Invierno & $-3,9$ & $-7,4$ & $-16,4$ & $-21,8$ & $-4,3$ & $-8,7$ & $-20,9$ & $-27,6$ \\
\hline Verano & 0,3 & 2,6 & 14,8 & 25,9 & 0,1 & 2,6 & 19,1 & 34,4 \\
\hline Primavera & $-3,3$ & $-6,9$ & $-16,1$ & $-21,2$ & $-4,1$ & $-8,2$ & $-19,3$ & $-25,2$ \\
\hline Otoño & $-2,5$ & $-4,7$ & $-9,4$ & $-11,0$ & $-3,3$ & $-6,1$ & $-12,1$ & $-14,0$ \\
\hline
\end{tabular}

B. Supuesto de un incremento de las temperaturas aparentes y medias diarias en dos grados

\begin{tabular}{|c|c|c|c|c|c|c|c|c|}
\hline & \multicolumn{4}{|c|}{ Ajuste con temperaturas aparentes } & \multicolumn{4}{|c|}{ Ajuste con temperaturas medias } \\
\hline & $\begin{array}{l}<65 \\
\text { años }\end{array}$ & $\begin{array}{c}65-74 \\
\text { años }\end{array}$ & $\begin{array}{c}75-84 \\
\text { años }\end{array}$ & $\begin{array}{l}>84 \\
\text { años }\end{array}$ & $\begin{array}{l}<65 \\
\text { años }\end{array}$ & $\begin{array}{l}\text { 65-74 } \\
\text { años }\end{array}$ & $\begin{array}{l}75-84 \\
\text { años }\end{array}$ & $\begin{array}{l}>84 \\
\text { años }\end{array}$ \\
\hline Total anual & $-17,9$ & $-30,8$ & $-47,4$ & $-45,9$ & $-21,8$ & $-37,6$ & $-53,7$ & $-47,0$ \\
\hline Invierno & $-7,7$ & $-15,0$ & $-33,7$ & $-44,9$ & $-8,7$ & $-17,8$ & $-41,7$ & $-57,4$ \\
\hline Verano & 1,0 & 6,5 & 34,6 & 58,9 & 1,1 & 7,3 & 45,8 & 80,7 \\
\hline Primavera & $-6,5$ & $-13,4$ & $-31,4$ & $-40,9$ & $-8,0$ & $-15,8$ & $-36,8$ & $-47,5$ \\
\hline Otoño & $-4,7$ & $-8,8$ & $-16,8$ & $-19,0$ & $-6,2$ & $-11,3$ & $-21,0$ & $-22,9$ \\
\hline
\end{tabular}

FUENTE: Elaboración propia a partir de series climáticas facilitadas por el Servei Meteorològic de Catalunya y datos sobre mortalidad proporcionados por la Direcció General de Recursos Sanitaris de la Generalitat de Catalunya

Los efectos de los posibles incrementos de las temperaturas previsiblemente afectarían especialmente a los grupos de edad avanzada que, por un lado se beneficiarían de una importante reducción de la mortalidad, sobre todo durante el invierno y la primavera (CUADRO 3). La disminución de la mortalidad en otoño sería sensiblemente menor, hasta el punto de suponer cantidades aproximadamente equivalentes o incluso, ligeramente inferiores a la mitad de las alcanzadas en invierno y primavera en los grupos de edad superior a 84 años y próximas, pero claramente menores que las calculadas para estas dos estaciones en los dos grupos de inferior edad. Las proporciones en cuanto a las diferencias de las mermas de la mortalidad en invierno y las dos estaciones intermedias serían similares en el supuesto de incrementos termométricos de uno o dos grados, si bien, como es esperable, según las estimaciones realizadas, el número de muertes que podría evitarse tras un incremento de dos grados, superaría ampliamente a las calculadas bajo el supuesto del incremento en un único grado que, como parece indudable, habría de producirse con antelación, de manera que las obtenidas para cada una de las 
estaciones de invierno, primavera y otoño con dos grados de aumento superarían ligeramente al doble de las que se evitarían bajo el aumento de un único grado de temperaturas aparentes o medias. En cambio, la mortalidad previsiblemente experimentaría un incremento reducido durante el verano entre las personas menores de 65 años y claramente apreciable en el grupo de personas de 65 a 74 años, sin que este aumento llegara a compensar la disminución del número de fallecimientos estimada para cada una de las restantes estaciones, mientras que en el grupo de 75 a 84 años el incremento de muertes en verano sería similar a la disminución estimada para invierno o primavera y duplicaría a la obtenida para otoño. Finalmente, en el grupo de las persones de mayores de 84 años, el aumento de la mortalidad sería totalmente superior a la reducción posible de cada una de las restantes estaciones. Los ajustes realizados en relación con las temperaturas máximas y mínimas diarias ofrecen resultados parecidos a los obtenidos con las temperaturas aparentes y medias diarias, por cuyo motivo se ha prescindido de su inclusión en la correspondiente tabla.

En resumen, el previsible y probable cambio climático, que muy bien podría concretarse hacia mediados del siglo presente en un incremento de las temperaturas del orden de 1 a $2^{\circ} \mathrm{C}$ en amplias áreas del este y nordeste de la península Ibérica, según las estimaciones anteriormente citadas, posiblemente permitiría una reducción de la mortalidad en Barcelona sobre todo durante las estaciones de invierno y primavera y, en menor medida durante el otoño. Por el contrario, el número de fallecimientos aumentaría notablemente durante los meses de verano. Las variaciones de reducción e incremento estacional de la mortalidad afectarían ostensiblemente de manera preferente a los grupos de personas mayores de 74 años, las cuales resultarían mucho más perjudicadas por el aumento de fallecimientos en verano. Este aumento castigaría en menor medida a las persones de 65 a 74 años y tan sólo ligeramente a los menores de 65 años.

Las estimaciones formuladas en relación a las posibles variaciones de los efectos sobre la mortalidad de las previsiones de cambio climático para los próximos decenios únicamente pretenden destacar precisamente la posibilidad y probabilidad de que no serán insignificantes. La medida en que las estimaciones propuestas lleguen a coincidir o a aproximarse a la evolución real de las proporciones de incremento o disminución de fallecidos en las distintas estaciones del año, como resulta obvio, no sólo dependerá de la bondad de los ajustes realizados, sino también de posibles impactos indirectos del propio cambio climático y, sobre todo, de las disponibilidades y medidas que se dispongan en cada momento destinadas a proteger la salud de las personas. Estas medidas no deberían limitarse a minimizar los efectos del calor 
que, por supuesto es preciso establecer, sino también reducir los impactos de los episodios fríos y del clima reinante durante el invierno y estaciones intermedias que, a pesar de los posibles beneficios que se deriven del incremento de las temperaturas, continuarán registrando con toda probabilidad unas tasas de mortalidad superiores a las del verano. Precisamente una reducción de la mortalidad durante los meses más fríos del año puede contribuir a un incremento de las defunciones provocadas o exacerbadas por las temperaturas extremada o relativamente más elevadas de los meses más cálidos, tal como han puesto de manifiesto trabajos anteriormente citados (RoCKLÖV et al., 2009; SUNYER, 2008). Por este motivo, el efecto hipotéticamente positivo que comportaría un incremento climático de las temperaturas de uno a dos grados, en tanto que la reducción de la mortalidad durante el invierno y, en menor medida durante la primavera y el otoño, podría compensar e incluso superar al aumento estimado para el verano, probablemente podría manifestarse menos evidente que los efectos nocivos ocasionados por el calor por cuanto los impactos de las temperaturas extremadamente elevadas, particularmente las olas de calor, se manifiestan a continuación del advenimiento de eventos de esta naturaleza o en los días inmediatos posteriores, mientras que los efectos de las olas de frío ordinariamente son menos drásticos tras su ocurrencia y persisten, aunque con menor intensidad, durante varios días, hasta dos semanas (CARDER et al., 2012). De manera que no parece arriesgado aceptar que son temperaturas relativamente bajas mantenidas durante los meses más fríos del año, más que episodios puntuales, las que provocan o exacerban la mortalidad entre las personas que forman parte de los denominados grupos de riesgo.

El progresivo envejecimiento de la población de Barcelona, Cataluña y la totalidad de España comportará un gradual incremento en el número de personas potencialmente más sensibles a los avatares atmosféricos y, sin duda, a la exacerbación de la mortalidad a lo largo de los veranos previsiblemente más cálidos que en tiempos pretéritos y presentes. Dada la limitación de recursos que en nuestro país sufren las personas tras la conclusión de su período de actividad laboral, será necesario prever toda una serie de medidas y actividades destinadas a proteger a todas las personas que carezcan de los medios y de la formación e información precisa para proteger su salud ante episodios atmosféricos extremos, no sólo de calor, cuyo acaecimiento en frecuencia e intensidad se espera irá en aumento, sino también frente a eventos de frío severo, que, a pesar del calentamiento anunciado, y de la consiguiente disminución de las tasas de mortalidad, particularmente en invierno, pero también en primavera y otoño, continuarán produciéndose y provocando impactos capaces de acelerar procesos patológicos capaces de acabar con la vi- 
da de no pocos pacientes crónicos, o de causar daños irreparables en los denominados grupos de riesgo.

\section{MITIGACIÓN Y ADAPTACIÓN AL CAMBIO CLIMÁTICO. LA NECESIDAD DE SISTEMAS DE ALERTA, AVISO Y SOPORTE A LA POBLACIÓN}

El cambio climático, con toda probabilidad, y, de acuerdo con el consenso prácticamente universal existente entre los científicos, comportará un incremento en la frecuencia e intensidad de las olas de calor así como en la reiteración de veranos más cálidos e inviernos más suaves. Los impactos en la salud de estas mutaciones, que previsiblemente se irán estableciendo, dependerá mucho de las condiciones y circunstancias de cada país, pero que, para algunos autores, serán principalmente negativos (GÓMEZ-ACEBO et al., 2010; MCMiCHAEL et al., 2006). Por más que, ciertamente, las tasas de mortalidad invernales de los países fríos y templados de Europa y América sobrepasan actualmente a las de verano (HAJAT et al., 2004; KEATINGE et al., 2000), razón por la cual parece del todo necesario el establecimiento de medidas destinadas a reducir los efectos del frío, sobre todo entre las personas de más edad (KEATINGE y DONALDSON, 2004), la mayor parte de los estudios publicados en Europa y los Estados Unidos acerca de la relación y posibles impactos de los avatares atmosféricos en la salud humana están dedicados a analizar los efectos provocados bajo episodios extremadamente cálidos. Todos estos trabajos destacan, además de la conocida relación entre las temperaturas y la morbilidad o, preferentemente, la mortalidad, la preocupación por el incremento de los posibles riesgos que el cambio climático pueda comportar, después que el calentamiento global ya haya empezado a afectar la salud, y la necesidad de adoptar estrategias de mitigación de este proceso y de adaptación ante un futuro clima más cálido. Sin negar los posibles efectos del cambio climático, DerByshiRE (2006) no cree que los efectos sean insuperables y confía con las actuales posibilidades tecnológicas y las que aporte una innovación continuada para hacer frente a los peligros que pueda comportar.

La mitigación del cambio climático, y especialmente de su proceso de calentamiento global, exigiría una reducción significativa de los gases de efecto invernadero. Las acciones encaminadas a la consecución de este objetivo aportarían, además de frenar o moderar el actual proceso de cambio climático, importantes beneficios para la salud, como han destacado la decena de artículos publicados el año 2009 en el número 374 de la revista The Lancet. Uno de estos trabajos subraya que la reducción de gases de efecto invernadero implicaría la toma y aplicación de decisiones en cuatro campos: producción de electricidad, transportes, producción de alimentos y uso doméstico 
(HAINES et al., 2009). Determinados cambios en la producción de electricidad, sobre todo la obtenida a partir de la combustión de carbón y petróleo, además de una reducción en la emisión de dióxido de carbono, habría de permitir también una disminución del vertido de partículas contaminantes a la atmósfera y, en consecuencia, beneficios importantes para la salud, especialmente en territorios donde la contaminación es muy elevada (WOODCOK et al., 2009). El transporte movido por energía procedente de la combustión de productos fósiles constituye otra fuente importante de gases de efecto invernadero y de partículas contaminantes, de manera que su reducción restringiría la presencia de estos gases en la atmósfera, algunos de los cuales, como los óxidos de nitrógeno, son nocivos para la salud, así como de partículas de tamaño inferior a 2,5 $\mu \mathrm{m}$, de efectos también nocivos para el organismo humano (MARKANDYA et al., 2009). La producción de alimentos supone igualmente el origen de una importante generación de gases de efecto invernadero, ya que la conversión de bosques en tierras de cultivo, los fertilizantes y el funcionamiento de la maquinaria agrícola generan dióxido de carbono y óxidos de nitrógeno, mientras que los restos de la producción agraria y la fermentación entérica de los alimentos consumidos por el ganado, principalmente el rumiante, liberan otro gas importante de efecto invernadero como es el metano (FRIEL et al., 2009; MCMICHAEL et al., 2007). Incluso la utilización de biomasa para la elaboración de pan o la satisfacción de las necesidades domésticas puede suponer un riesgo para la salud debido a la generación de gases de efecto invernadero, monóxido y dióxido de carbono principalmente, y partículas, que serán particularmente agresivas para los que trabajan en panaderías o en cocinas donde se consumen estos combustibles (WILKINSON et al., 2009). Las combustiones para la producción de energía, transporte o uso doméstico, además de dióxido de carbono y metano, generan sulfatos y aerosoles de carbono orgánico, unos y otros nocivos para la salud. Las susodichas combustiones, además de verter a la atmósfera óxidos de nitrógeno e hidrocarburos, propician la formación de un gas de vida corta, resultante de complejas reacciones a partir de productos volátiles de las propias combustiones, el ozono, dañino sobre todo para el aparato respiratorio (SMITH et al., 2009). Resulta indudable que las acciones necesarias para que, con la tecnología actual, pueda conseguirse tan sólo moderar significativamente el cambio climático, exigirían la transmutación de nuestro estilo de vida. Tendríamos que conducir menos, volar menos y alimentarnos de manera bien diferente. Es por este motivo que los responsables políticos se resisten a pedir a los ciudadanos unas transformaciones que podrían ser percibidas como un retroceso en el desarrollo humano, y más durante la inseguridad financiera de los últimos años (HORTON, 2009; LiM et al., 2009). Las indecisiones o dificultades que habrían de afrontar las autoridades para defender unos acuerdos de no fácil aceptación radican, primeramente, en los 
sacrificios o incomodidades que comportaría adoptar nuevos hábitos y renunciar a unas costumbres fuertemente arraigadas, pero también en el desconocimiento de los beneficios que se derivarían para la salud de unas acciones de mitigación del cambio climático como las indicadas.

$\mathrm{Si}$, tal como se ha destacado y es obvio, resulta difícil, casi imposible, llegar actualmente a un compromiso real que implique a todos los países del Mundo en la reducción de la generación de gases capaces de intensificar el cambio climático, tampoco sería fácil conseguir la aceptación de medidas paliativas por parte de un limitado número de estados por razones económicas. En efecto, la aplicación por parte de unos pocos significaría un esfuerzo escasamente apreciable en el proceso de cambio climático y una pérdida económica para aquellos que aceptasen el reto. Los beneficios que aportaría una reducción de las emisiones de gases de efecto invernadero están fuera de duda y parece ilusoria la confianza de GoKLANY (2009a; 2009b) en que las actuales y futuras posibilidades técnicas y económicas de adaptación aporten más beneficios y mejor precio que la mitigación del propio cambio climático. En contra de esta opinión, las políticas firmes y determinantes de mitigación del cambio climático podrían ofrecer inmediatos y positivos efectos en la salud de la población (MCMichAEL et al., 2009). La renuncia a toda mitigación del cambio climático no puede justificarse en razones estrictamente económicas, porque los beneficios para la salud, aunque insuficientemente conocidos (HORTON, 2009), que supondría una reducción de las emisiones habrían de permitir un ahorro en la financiación de servicios sanitarios superior a los gastos de muchas políticas de mitigación (CAMPBELL-LENDRUM et al., 2009). En todo caso, es necesario entender que el cambio climático es el precio que debemos pagar por la aceptación de políticas avarientas (CHAKUROVA e IVANOV, 2005) y, más que una cuestión de acuerdos internacionales o de disponibilidades económicas, se trata de decidir en qué Mundo queremos vivir. La necesidad y urgencia de tomar medidas dirigidas a proteger la salud es tan incuestionable que constituiría un riesgo no hacer nada (GILL y STOTT, 2009).

Además de un aumento de la frecuencia e intensidad de las olas de calor, es previsible un incremento de la vulnerabilidad de los efectos del cambio climático durante el presente siglo, debido al envejecimiento de la población, particularmente en los países más desarrollados, y al progresivo y rápido crecimiento de las ciudades y, en consecuencia, del número de personas sometidas a los efectos de isla de calor urbana que han de padecer los habitantes de los centros de las grandes ciudades, como es el caso de Barcelona, unos impactos del calor en su salud superiores a los que han de soportar los 
que viven en áreas rurales o suburbanas (CONTI et al., 2005; TAN et al., 2010).

En definitiva, los beneficios que reportaría una decidida y eficaz mitigación del cambio climático dentro de las líneas señaladas están fuera de dudas. Además, los esfuerzos que requeriría la financiación de las distintas acciones de mitigación habrían de representar inversiones rentables a no muy largo plazo. Sin embargo, su viabilidad requeriría un acuerdo de universal aceptación que no parece posible en la actualidad, por cuyo motivo es del todo necesario que los responsables de la administración pública arbitren medidas destinadas a minimizar los impactos de los avatares atmosféricos extremos.

Ante las dificultades existentes para establecer compromisos de general aceptación que permitan una mitigación del cambio climático, se impone la adopción de toda una serie de acciones capaces de reducir los posibles efectos negativos. Uno de los métodos más adecuados, si no el que más, para minimizar los efectos provocados por el incremento de las temperaturas y episodios excepcionalmente cálidos, consiste en el establecimiento de un sistema de alerta, aviso y soporte a la población ante el advenimiento de situaciones atmosféricas de riesgo para la salud.

El sistema de alerta y aviso a la población de Philadelphia ante tipos de tiempo cálidos susceptibles de afectar la salud propuesto por KALKSTEIN et al. (1996) fue pionero en el posterior establecimiento de soluciones parecidas en otras ciudades de todo el Mundo. El sistema prevé la intervención de los responsables políticos de la ciudad y otros estamentos. La televisión, la radio y la prensa se encargan de informar a la población de la inminencia o presencia de condiciones opresivas del tiempo y de la manera de evitar o minimizar los efectos nocivos provocados por el calor en su organismo. Los propios medios de difusión incitan a que amigos, vecinos y otros voluntarios hagan visitas diarias a las personas de edad avanzada que viven solas y cuiden de que estas personas cuenten con suficientes elementos y servicios para protegerse de los tipos de tiempo que representan riesgos para la salud. La corporación municipal abre una línea telefónica especial, el número de la cual aparece en los medios de comunicación y pantallas en el centro de la ciudad, destinada a informar y aconsejar al público en general para evitar los daños provocados por el estrés térmico. El departamento de salud dispone o contrata personal para informar y ayudar a quienes requieran cuidados especiales. Además, las compañías de servicios, particularmente las encargadas del suministro de agua y electricidad, han de garantizar el mantenimiento de sus actividades mientras persista el período de emergencia. Al mismo tiempo, el servicio de urgencias médicas incrementa su personal en previsión de 
un aumento de la demanda. El sistema de alerta y aviso a la población de Philadelphia diseñado por Kalkstein permitió la preservación de un número de vidas estimado en 2,6 por cada día excepcionalmente caluroso y los tres siguientes, de manera que, en tres años, se llegaron a evitar 117 muertes (EBI et al., 2004).

A pesar de la eficacia evidente de este sistema, tan sólo dos ciudades europeas, Lisboa y Roma, disponían de un conjunto de disposiciones y recursos con objetivos parecidos antes del año 2003, cuando buena parte del continente padeció la consabida ola de calor de excepcional intensidad. Pero este mismo año, las ciudades de Turín, Milán y Bolonia inauguraron sus propios sistemas, mientras buen número de ciudades de Estados Unidos contaban con este servicio y proyectaban su instalación en todos los núcleos poblacionales con más de 500.000 habitantes, y dos ciudades canadienses más la china de Shanghái habían inaugurado los suyos (SHERIDAN y KALKSTEIN, 2004).

A raíz de los impactos de las olas de calor de 2003, los países de Europa desarrollaron con cierta celeridad sistemas de alerta y aviso, mientras un apreciable número de ciudades de los Estados Unidos todavía no contaban con un plan de respuesta a situaciones meteorológicas extremas o únicamente preveían disposiciones de alcance limitado y carentes de eficacia (BERNARD y MCGEEHIN, 2004). Francia estableció un sistema de vigilancia y advertencia ante la inminencia de olas de calor basado en las previsiones meteorológicas de sobrepasar determinados umbrales termométricos, que incluía, además de una amplia difusión de los posibles riesgos en los medios de difusión social, la emisión de una serie de disposiciones dirigidas a garantizar el mantenimiento de los servicios de distribución de agua y energía, así como de los servicios médicos y sanitarios, ante el previsible aumento de la demanda, y a organizar medidas de soporte social para las personas que potencialmente puedan necesitarlo (PASCAL et al., 2006). Esto permitió una reducción de la mortalidad durante la ola de calor del año 2006 estimada en torno a 4.400 personas en relación a la esperada (FouILLET et al., 2006). La capacidad de las olas de calor de provocar efectos inmediatos, sobre todo entre personas con antecedentes cardiovasculares, incluso con resultado de muerte antes de su ingreso en un centro hospitalario (LiNARES y DíAZ, 2007), justifican la necesidad de que los sistemas de emergencia y soporte a la población dispongan de medios suficientes para atender a las personas que forman parte de los grupos de más alto riesgo. La intervención de 2.550 practitioners o ayudantes sanitarios en un programa de atención a un grupo de estas características que representaba el $5 \%$ de las personas mayores de 65 años de Roma durante el verano de 2007, principalmente dirigido a las de 
75 y más años, permitió una reducción significativa de la mortalidad entre las que participaron en el programa, las cuales padecieron un exceso de mortalidad del $7 \%$, mientras que fue del $13 \%$ entre las que no participaron (BARGAGLI et al., 2007).

El Departament de Salut i Medi Ambient de la Generalitat de Catalunya, en colaboración con Servei Meterològic de Catalunya (SMC), puso en marcha un plan para prevenir los efectos de las olas de calor sobre la salud (POCS), contando con la participación de otros departamentos del gobierno autónomo y entidades municipales y sanitarias. El POCS fue diseñado con tres objetivos esenciales: predecir, con la máxima antelación que permitan los medios técnicos las posibles situaciones atmosféricas de riesgo, coordinar los medios y recursos disponibles para hacer frente a inminentes olas de calor y minimizar los impactos del calor en la salud de la población, particularmente de las personas más vulnerables. El plan se aplica desde el día 1 de junio hasta el 15 de septiembre y se activa cuando el SMC prevé un incremento de la temperatura máxima diaria por encima del $98^{\circ}$ percentil, el valor del cual se cifra en $33,1^{\circ} \mathrm{C}$ en Barcelona, $32,1^{\circ} \mathrm{C}$ en Tarragona , $37,5^{\circ} \mathrm{C}$ en Lleida y $36,1^{\circ} \mathrm{C}$ en Girona. Cuando esto sucede, el SMC emite una situación meteorológica de peligro de nivel 1 que alcanzará el nivel 2 si prevé que el citado percentil será superado durante tres o más días. Los cálculos para estas previsiones han de realizarse en relación a 10 municipios representativos de diferentes puntos del litoral por un lado, y del interior del territorio por otro, y es posible su activación y desactivación para cada uno de estos sectores por separado. Una amplia tarea de información a la población sobre los riesgos de las temperaturas excepcionalmente altas para la salud de la población marca el inicio de cada campaña anual, consistente en la edición y distribución de los centros sanitarios y oficinas de farmacia de un folleto que incluye recomendaciones válidas para toda la población. Además, se prevé para cada centro de atención primaria, hospital y residencia geriátrica de un plan de actuación que dispense ayuda a las personas que la requieran, el cual incluye un censo actualizado de las personas con mayor riego que es preciso contrastar con los servicios sociales. Al mismo tiempo, se hace un seguimiento diario de los datos facilitados por el SMC y de las defunciones en los diez municipios objeto de control. A partir del 15 de junio, se procede a hacer una divulgación semanal de la evolución de las temperaturas y defunciones, así como de las recomendaciones a las personas más frágiles y con alto riesgo social y se abre una línea telefónica para facilitar información a quien la solicite. Una situación de riesgo de nivel 2 requiere la movilización de recursos de emergencia de diferentes estamentos o servicios de la Generalitat y de la Cruz Roja. 
La experiencia acumulada en el establecimiento y evaluación de sistemas de alerta y atención en buen número de países de todo el Mundo han aportado más que un notable progreso en el conocimiento de los efectos de las condiciones atmosféricas extremas sobre la salud y en la manera de reducir sus impactos más graves, pero, lejos de ofrecer una formulación de métodos y procedimientos de aplicación universal, han abundado en destacar la diversidad de las realidades climáticas existentes, así como de las sociedades que ocupan los diferentes espacios, unos climas en proceso de mutación y unas sociedades cambiantes demográfica, social y culturalmente. Esto exige una continuidad en las tareas de evaluación de los resultados conseguidos año tras año y la introducción de las modificaciones que se planteen a la vista de las experiencias acumuladas. El simple envejecimiento de la población puede requerir un ajuste de los umbrales termométricos que determinen la activación de los sistemas adoptados en cada territorio, hasta el punto que sería aconsejable regularizarlos al menos cada diez años (MIRON et al., 2008). A pesar de las diferencias existentes entre los sistemas de alerta y asistencia a la población frente a los episodios de calor extrema que han sido implantados durante los últimos años, las experiencias resultantes de su aplicación constituyen unas bases de información y referencias valiosas en la proyección de nuevos sistemas con este mismo objetivo, así como en el momento de proceder a su evaluación y calibración o mejora. El plan establecido en Barcelona y el resto de Cataluña después de las olas de calor de 2003 todavía no ha podido ser evaluado y calibrado tras su aplicación en olas de calor de intensidad parecida a las padecidas aquel año, ya que, desde entonces, no han sucedido fenómenos de características similares. Su eficacia, dado que las previsiones meteorológicas son generalmente y cada vez más fiables, dependerá sobre todo de los medios humanos y sanitarios que estén en disposición de actuar cuando vuelvan a suceder episodios parecidos, que previsiblemente no faltarán en veranos futuros. Las previsiones de llegada de masas de aire estresantes, portadoras de olas de calor, por más que sean ordinariamente fiables, requieren un seguimiento continuo de la evolución de las temperaturas y de la humedad lo cual habrá de permitir una estimación de los hipotéticos excesos de mortalidad y activar las medidas para su minimización. Para que esto sea posible, las autoridades responsables de activar el sistema, además de cuidar de su amplia difusión y poner en marcha los protocolos de asistencia a la población, deberían tener en cuenta la diversidad de las características demográficas, sociales y económicas, cambiantes con el tiempo. En cuanto a los grupos de mayor riesgo, además de los ancianos, un reciente trabajo ha destacado que, en Barcelona, las mujeres son más sensibles a los efectos del calor que los hombres y los niveles más bajos de educación también suponen un riesgo (BORRELL et al., 2006). La mayor susceptibilidad de las mujeres puede explicarse, al menos en parte, porque su vida 
media es más prolongada que la de los hombres y, por lo tanto, su proporción entre las personas de más edad es más elevada (GARCÍA-HERRERA et al., 2005). El plan debería incluir información sobre las personas de edad avanzada que viven solas o en condiciones precarias, particularmente si padecen alguna afección cardiovascular o respiratoria de carácter crónico y elaborar un archivo de candidatos a una atención personalizada.

En definitiva, un sistema de alerta y ayuda a la población, para que sea eficaz implica una amplia dedicación de medios y, en consecuencia, exige importantes recursos económicos, pero su implantación no reviste gran complejidad ni el dispendio de mucho tiempo. Más difícil, e incluso en buena parte inviable, sería una deseable acomodación de las viviendas y el entorno urbano en orden a reducir los impactos del calor extremo. La construcción de viviendas óptimamente orientadas, con mejores materiales y tecnológicamente preparadas para reducir las respuestas a las condiciones atmosféricas cambiantes requiere unas disponibilidades económicas difíciles de asumir en obras nuevas, en tanto que es impensable la adaptación o substitución de la totalidad de las edificaciones existentes. Las actuales normas de edificación, sobre todo en las grandes ciudades como Barcelona, ya tienen en cuenta, al menos formalmente, la necesidad de utilizar materiales y técnicas de aislamiento, pero su adopción es deficiente. La obtención de energía solar a partir de placas instaladas en el tejado o cobertura de la casas no se aplica con suficiente rigor, y cada vez es más frecuente la instalación de aire acondicionado, con los consiguientes problemas que su generalización significaría para el cambio climático, debido al gran incremento en el consumo de electricidad que, a su vez, comporta la generación de gases de efecto invernadero y contaminantes nocivos para la salud. Una medida mínimamente efectiva para la mitigación y adaptación al cambio climático sería cuidar con esmero la extensión y mantenimiento de arbolado en las ciudades. Además de la pequeña reducción de los impactos de la radiación sobre el suelo y consiguiente moderación de las temperaturas y absorción de dióxido de carbono, con una oportuna campaña de difusión podría representar un motivo de preocupación de la población por los efectos del cambio climático en la salud.

El estudio de las posibles consecuencias del cambio climático para la salud todavía se encuentra en su infancia (VIGOTTI y MugGeo, 2006), por lo que merece la dedicación de los esfuerzos que sean precisos para mejorar progresivamente su conocimiento y conseguir sistemas de alerta y ayuda más eficaces, aprendiendo de las propias experiencias y de las conocidas de otros territorios. Tal como subrayan KOVATS y JENDRITZKY, 2006, los países europeos necesitan aprender unos de otros. 


\section{CONCLUSIÓN}

Por más que sea altamente difícil prever el alcance de los efectos del denominado cambio climático sobre la salud humana, parece altamente probable que los riesgos más preocupantes serán los derivados del progresivo calentamiento medioambiental, sobre todo del incremento de los episodios de calor extremo. Estos riesgos no pueden obviarse porque un moderado ascenso climático de las temperaturas favorezca una disminución de la mortalidad durante los meses más fríos, capaz de contrarrestar los efectos nocivos del calor extremo, hasta el punto de suponer una posible reducción de la mortalidad total anual relacionada con la evolución de la temperatura del aire. Por otro lado, la reducción de la mortalidad en invierno beneficiaría básicamente a las personas que constituyen los consabidos grupos de riesgo, esencialmente enfermos crónicos y mayores de 75 años, parte de las cuales muy probablemente podrían haber fallecido durante el verano precedente o morirían en el siguiente. Por todo ello, resulta altamente conveniente o, mejor, necesario dotar de más medios a los actuales sistemas de alerta y aviso ante la proximidad o inminencia de olas de calor. Se trataría, por un lado, y en primer lugar, de optimizar recursos existentes, como son las previsiones meteorológicas, cada vez más precisas y acertadas y de una mayor difusión de la información a través de los medios de comunicación social y en los centros sanitarios y geriátricos. Por otra parte, un sistema eficaz debería prever el soporte a las personas de más riesgo, especialmente a las de mayor edad que viven solas y en condiciones deficientes, lo cual requeriría contar con un equipo de colaboradores sanitarios dedicados a informar y atender a estas personas. Igualmente, sería deseable la acomodación de los edificios y equipamiento de los hogares para hacer frente a situaciones atmosféricas adversas, algo de muy difícil o imposible ejecución a corto plazo y, en todo caso, condicionado por las disponibilidades económicas. Por último es preciso no olvidar que, a pesar del calentamiento, continuarán produciéndose, posiblemente con menos frecuencia, episodios y olas de frío merecedoras de arbitrar medidas de aviso y protección destinado en general a las mismas personas que requieren ayuda ante eventos de calor extremo.

\section{AGRADECIMIENTOS}

El trabajo se encuadra en el marco del Grupo de Climatología 2009 SGR 443 (Generalitat de Catalunya) y, metodológicamente, en el proyecto CGL2011-29263-C02-01 (Ministerio de Economía y Competitividad). Nuestro agradecimiento al Servei Meteorològic de Catalunya por la cesión de datos de la estación meteorológica de Fabra y al Servei d'Informació i Estudis del Departament de Salut de la Generalitat de Catalunya por haber proporcionado datos sobre mortalidad registrados en Barcelona. 


\section{BIBLIOGRAFÍA}

Allen, M. R.; Framec, D. J.; Huntingford, C.; Jopnes, C. D.; Lowe, J. A.; MEINSHAUSEN, M. et al. (2009): «Warning caused by cumulative carbon emissions towards the trillionth tone». Nature, 458, 1163-1166.

Anderson, B. G.; BELL, M. L. (2009): «Weather-related mortality in the United States». Epidemiology, 20 (2), 205-213.

Baccini, M.; Biggeri, A.; ACcetta, G.; Kosatsky, T.; Katsouyanni, K.; ANALITIS, A. et al. (2008): «Heat effects on mortality in 15 European cities». Eidemiology, 19 (5), 711-719.

BALLESTER, F. (1996): «Meteorología y salud. La relación entre la temperatura ambiental y la mortalidad». Revista Española de Salud Pública, 70, 251-259.

Bargagli, A.; Michelozzi, P.; Manuela, D.; Chierchini, P.; CASAli, V.; CERVELLI, S. et al. (2007): «Summer 2007 heat waves in Roma: results of the Prevention Programme based on General Practitioner Surveillance». Epidemiology, 19 (6 Suppl.).

BASU, R.; FENG, W. Y.; OSTRO, B. D. (2008): «Characterizing temperature and mortality in nine California counties». Epidemiology, 19(1), 138-145.

Bell, M. L.; O'NEILl, M. S.; RANJIT, N.; BORJA-ABURTO, V. H.; CiKfuENTES, L. A.; GOUVEIA, N. C. (2008): «Vulnerability to heat-related mortality in Latin America: a case-crossover study in Sao Paulo, Brazil, Santiago, Chile and Mexico City, Mexico». International Journal of Epidemiology, 37 (August), 796-804.

BERNARD, S. M.; MCGEEHIN, M. A. (2004): «Municipal heat wave response plans». American Journal of Public Health, 94(9), 1520-1521.

Borrell, C.; Marí-Dell'Olmo, M.; RodríGuez-SAnZ, M.; García-Olalla, P.; CAYLÁ, J. A.; BENACH, J. et al. (2006): «Socioeconomic position and excess during the heat wave of 2003 in Barcelona». European Journal of Epidemiology, 21, 633-640.

Brunet, M.; Casado, M. J.; De Castro, M.; Galán, P.; LÓPez, J. A.; Martín, J. M. et al. (2009): Generación de escenarios regionalizados de cambio climático para España Madrid: Agencia Estatal de Meteorología.

Calbó, J.; SÁnChez-Lorenzo, A.; CunillerA; J.; BARrerA, A. (2010): «Projeccions i escenaris de futur». In J. E. LLEBOT (Ed.): Segon informe del canvi climàtic a Catalunya. Barcelona: Generalitat de Catalunya. Institut d'Estudis Catalans, 183-239

CampBell-Lendrum, D.; Bertollini, R.; Neira, M.; Mcmichael, A. (2009): «Health and climate change: a roadmap for applied research». Lancet, 373 (9676), 1663-1665.

Carder, M.; Mcnamee, R.; Beverland, I.; Elton, R. A.; Cohen, G. R.; Boyd, J.; AgIUS, R. M. (2012): «The lagged effect of cold temperature and wind chill on cardiorespiratory mortality in Scotland». Occupational and Environmental Medicine, 2005(62), 702-7010.

Conti, S.; Meli, P.; Minelli, G.; Slimini, R.; Toccaceli, V.; Vichi, M. et al. (2005): «Epidemiologic study of mortality during the Summer 2003 heat wave in Italy». Environment Research, 98, 390-399. 
CURRIE, B. J.; JACUPS; S. P. (2003): «Intensity of Rainfall and Severity of Melioidosis, Australia». Emerging Infectious Diseases, 9(12), 1531-1542.

Curriero, F. C.; Heiner, K. S.; SAMET, J. M.; Zeger, S. L.; StRUG, L.; PATZ, J. A. (2002): «Temperature and mortality in 11 cities of the Eastern United States». American Journal of Epidemiology, 155(1), 80-87.

CHAKUROVA, R.; IVANOV, L. (2005): «Extreme weather events in Bulgaria for the period 2001-03 and responses to address them». In W. KIRCH, B. MENNE; R. BERTOLLINI (Eds.): Extreme weather events and public health responses. Dresden: Springer, 337-242

Changnon, S. A.; KunKel, K. E.; ReInKE, B. C. (1996): «Impacts and responses to the 1995 heat wave: a call to action». Bulletin of the American Meteorological Society, 77(7), 1497-1506.

DERBYSHIRE, S. W. (2006): «Climate change and health». Lancet, 367 (9527), 1976.

DESSAI, S. (2002): «Heat stress and mortality in Lisbon Part I”. model construction and validation». International Journal of Biometeorology, 47, 6-12.

DíAZ, J.; LinARES, C.; GARCÍA-HERRERA, R. (2005): «Impacto de las temperaturas extremas en la salud pública: futuras actuaciones». Revista Española de Salud Pública, 79, 145-147.

EBI, K. L.; EXUZIDES, K. A.; LAU, E.; KELSH, M. (2004): «Weather changes associated with hospitalizations for cardiovascular diseases and stroke in California, 1983-1998». International Journal of Biometeorology, 49, 48-58.

EUROWINTER GROUP (1997): «Cold exposure and winter mortality from ischaemic heart disease, cerebrovascular disease, respiratory disease, and all causes in warm and cold regions of Europe». Lancet, 349 (9062), 1341-1346.

Fouillet, A.; Rey, G.; Laurent, F.; PAvillon, G.; Bellec, S.; Gujihenneuc, C. et al. (2006): «Excess mortality related to the august heat wave in France». International Archive Occupational Environmental Health, 80, 16-24.

Friel, S.; Dangour, A. D.; Garnet, T.; LOCK, K.; Chalabi, Z.; RoBerts, I. B. A. et al. (2009): «Public health benefits of strategies to reduce greenhouse-gas emissions: food and agriculture». Lancet, 374 (9706), 2016-2025.

GarcíA-Herrera, R.; DÍAZ, J.; Trigo, R. M.; HernándeZ, E. (2005): «Extreme summer temperatures in Iberia: health impacts and associated synoptic conditions». Annales Geophysicae, 23, 239-251.

GiLl, M.; STOTT, R. (2009): "Health professionals must act to tackle climate change». Lancet; 374 (9706), 1953-1955.

GOKLANY, I. M. (2009a): «Is the climate change the "Defining challenger of our age"?» Energy and Environment, 20(3), 279-302.

GOKLANY, I. M. (2009b): «Climate change is not the biggest global health threat». Lancet, 374 (9694), 973-974.

GómEZ-ACEBO, I.; DiERSSEN-SOTOS, T.; LLORCA, J. (2010): «Effect of cold temperatures on mortality in Cantabria (Northern Spain): A case-crossover study». $\mathrm{Pu}$ blic Health, 124, 398-403.

Haines, A.; MCMichael, A. J.; Roberts, I.; WoOdCOCK, J.; Markandya, A.; Armstrong, B. G.; CAmpbell-Lendrum, D.; Dangour, A. D.; Davies, M.; TONNE, C.; BARRET, M,; WiLKINSON, P. (2009): «Public health benefits of stra- 
tegies to reduce greenhouse-gas emissions: overview and implications for policy makers». Lancet, 374 (9707), 2104-2114.

HAJAT, S.; BIRD, W.; HAINES, A. (2004): «Cold weather and GP consultations for respiratory conditions by elderly people in 16 locations in the UK». European Journal of Epidemiology; 19, 959-968.

HALES, S.; EDWARDS, S. J.; KOVATS, R. S. (2003): «Impacts on health of climate extremes». In A. J. MCMICHAEL, D. H. CHAMPBELl-LENDRUM; C. F. CORVAlAN; K. L. EBI; A. GiTheKO; J. D. SCherAGA; A. WOODWARD (Eds.): Climate change and human health (pp. 79-102). Geneva, World Health Organization.

HORTON, R. (2009): «The climate dividend». Lancet, 374 (9705), 1869-1870.

Kaiser, R.; Rubin, C.; Henderson, A.; Alden, K.; Wolfe, M.; KieszaK, S.; PARROT, C.; ADCOCK, M. (2001): «Heat-related death and mental illness during the 1999 Cincinnati heat wave». The American Journal of Forensic Medicine and Pathology, 22(3), 303-307.

KALKSTEIN, L. S. (1993): «Health and climate change. Direct impacts in cities». Lancet, 342 (8884), 1397-1399.

Kalkstein, L. S.; Jamason, P. F.; Greene, J. S.; LibBy, J.; Robinson, L. (1996): «The Philadelphia hot weather-health watch/warning system: development and application, summer 1995». Bulletin of the American Meteorological Society, 77(7), 1519-1528.

KALKSTEIN, L. S.; VALIMONT, K. M. (1986): «An evaluation of Summer Discomfort in the United States Using a Relative Climatological Index». Bulletin of the American Meteorological Society, 67, 842-848.

KARL, T. R.; KNIGHT, R. W. (1997): «The 1995 Chicago heat wave: how likely is a recurrence?» Bulletin of the American Meteorological Society, 78(6), 1107-1119.

Katsouyanni, K.; TrichopoulOS, D.; ZaviTSANOS, X.; TOUlOMI, G. (1988): «The 1987 Athens Heatwave». Lancet, 332(8610), 573.

KEATINGE, W. R.; DonALDSON, G. C. (2004): «Winter mortality in elderly people in Britain». British Medical Journal, 329, 976.

Keatinge, W. R.; Donaldson, G. C.; Cordioli, E.; Martinelli, M.; Kunst, A. E.; MACKENBACH, J. P.; NAYHA, S.; VUORI, I. (2000): «Heat related mortality in warm and cold regions of Europe: observational study». British Medical Journal, 321, 670-673.

KellermanN, A. L.; TodD, K. H. (1996): «Killing Heat». The New England Journal of Medicine, 335(2), 126-127.

Kloner, R. A.; PoOle, K.; PerritT, R. L. (1999): «When throughout the year Is coronary death most Likely to Occur?». Circulation, 100, 1630-1634.

Kovats, R. S.; JendRITZKY, G. (2006): «Heat-Waves And Human Health». In B. MENNE; K. L. EBI (Eds.): Climate change and adaptation strategies for human health (pp. 63-97). Darmstadt: Springer.

KUMAR, S. (1998): «India's heatwave and rains result in massive death toll». Lancet, 351 (9119), 1869-1869.

Kunkel, K. E.; Changnon, S. A.; ReInke, B.; ARritT, R. W. (1996): «The July 1995 heat wave in the Midwest: a climatic perspective and critical weather factors». Bulletin of the American Meteorological Society, 77(7), 1507-1518. 
Lim, V.; Stubbs, J. W.; Nahar, N.; AMARAsena, N.; Chasudry, Z. U.; Mayosi, B.; VAN Der SpUY, Z.; Liang, R.; Neng, K.; METZ, G.; ClarK. G.; Williams, B.; Douglas, N.; Donohoe, J.; DanrchaiviJir, S.; COKer. P.; GILmore, I. (2009): «Politicians must heed health effects of climate change». Lancet, 374 (9694), 973.

LINARES, C.; DÍAZ, J. (2007): «Impact of high temperatures on hospital admissions comparative analysis with previous studies about mortality (Madrid)». European Journal of Public Health, 18(3), 317-322.

MaKiE, T.; HARADA, M.; KinUKAWA, N.; TOYOSHIBA, H.; YAMANAKA, T.; NAKAMURA, T.; SAKAMOTO, M.; NOSE, Y. (2002): «Association of meteorological and day-of-the-week factors with emergency hospital admissions in Fukuoka», Japan. International Journal of Biometeorology, 46, 38-41.

Markandya, A.; Armstrong, B. G.; Hales, S.; Chiabai, A.; Criqui, P.; Mima, S.; TONNE, C.; WILKINSON, P. (2009): «Public health benefits of strategies to reduce greenhouse-gas emissions: low-carbon electricity generation». Lancet, 374 (9706), 2006-2014.

MARTÍNEZ-NAVARRO, F.; SIMÓN-SORIA, F.; LÓPEZ ABENTE, G. (2004): «Valoración del impacto de la ola de calor del verano de 2003 sobre la mortalidad». Gaceta Sanitaria, 18(supl 1), 250-258.

MCMichael, A. J.; NeIRA, M.; Bertollini, R.; CAMPBll-LendRUM, D.; Hales, S. (2009): "Climate change: a time of need and opportunity for the health sector». Lancet, 374 (9707), 2123-2125.

McMichael, A. J.; NeIRA, M.; HeymanN, D. L. (2008a): «World Health Assembly 2008: climate change and health». Lancet, 371 (9628), 1895-1896.

McMichael, A. J.; Powles, J. W.; Butler, C. B.; UAuY, R. (2007): «Food, livestock production, energy, climate change, and health». Lancet, 370 (9594), 12531263.

McMichael, A. J.; Wilkinson, P.; Kovats, R. S.; Pattenden, S.; Hajat, S.; ARMSTRONG, B.; VAJANAPOOM, N.; NiCIU, E. M.; MOHOMED, N.; KinGKEOW, C.; KOSNIK, M.; O'NeILl, M. S.; RoMieU, I.; RAMÍREZ-Aguilar, M.; BARreto, M. L.; GoUveiA, N.; NiKIFOROV, B. (2008b): «International study of temperature, heat and urban mortality: the 'ISOTHURM' project». International Journal of Epidemiology, 37, 1121-1131.

McMichael, A. J.; WoOdRufF, R. E.; Hales, S. (2006): «Climate change and human health: present and future risks». Lancet, 367(9513), 859-869.

MEDINA-RAMÓN, M.; SCHWARTZ, J. (2007): «Temperature, temperature extremes, and mortality: a study of acclimatization and effect modification in 50 US cities». Occupational and Environmental Medicine, 64, 827-833.

Meinshausen, M.; Meinshausen, N.; Hare, W.; Raper, S. C. B.; Frieler, K.; KNUTTI, R. (2009): «Greenhouse-gas emission targets for limiting global warning to $2^{\circ} \mathrm{C} »$. Nature, 458, 1158-1162.

Michelozzi, P.; De Donato, F. K.; BARGAGLi, A. M.; D'IPPOliti, D.; DE SARIO, M.; MARINO, C.; SCHIFANO, P.; CAPPAI, G.; LEONE, M.; KIRCHMAYER, U.; VENTURA, M.; Di Gennaro, M.; Leonardi, M.; Oleari, F.; De Martino, A.; Perucci, C. A. (2010): «Surveillance of summer mortality and preparedness to reduce the 
health impact of heat waves in Italy». International Journal of Environmental Research and Public Health, 7, 2256-2273.

Mirón, I. J.; Criado, J. J.; DÍAZ, J.; LinARES, C.; MAYORAL, S.; MonTero, J. C. (2008): «Time trends in minimum mortality temperatures in Castile-La Mancha (Central Spain): 1975-2003». International Journal of Biometeorology, 52, 291299.

Naughton, M. P.; Henderson, A.; Mirabelli, M. C.; Kaiser, R.; Wilhelm, J. L.; KIESZAK, S. M. RuBIN, C. H.; MCGEEHIN, M. A. (2002): «Heat-related mortality during a 1999 heat wave in Chicago». American Journal of Preventive Medicine, 22(4), 221-227.

O'Neill, M. S.; Hajay, S.; ZanobetTI, A.; RamíreZ, M.; SchwartZ, J. (2005): «Impact of control for air pollution and respiratory epidemics on the estimated associations of temperature and daily mortality». International Journal of Biometeorology, 50, 121-129.

O'Neill, M. S.; ZanOBetTi, A.; SChWARTZ, J. (2003): «Modifiers of the temperature and mortality association in seven US cities». American Journal of Epidemiology, 157(12), 1074-1082.

Pajares, M. S.; DíAZ, J.; Montero, J. C.; Alberdi, J. C.; Mirón, I. (1997): «Mortalidad diaria en la comunidad de Madrid (1986-1991) para el grupo de 45 a 64 años: su relación con la temperatura del aire». Revista Española de Salud Pública, 71(2), 149-160.

PALECKI, M. A.; ChAngnon, S. A.; KunKel, K. E. (2002): «The nature and impacts of the july 1999 heat wave in the Midwestern United States: Learning from lessons of 1995». Bulletin of the American Meteorological Society; 82(7), 13531367.

PAN, W. H.; LI, L.A.; TSAI, M. J. (1995): «Temperature extremes and mortality from coronary heart disease and cerebral infarction in elderly Chinese». Lancet, 345(8946), 353-355.

PASCAL, M.; LAAIDI, K.; LEDRANS, M.; BAFFERT, E.; CASERIO-SCHÖNEMANN, C.; LE Tertre, A.; Manach, J.; Medina, S.; Rudant, J.; Empereur-Bissonnet, P. (2006): «France's heat health watch warning system». International Journal of Biometeorology, 50, 144-153.

PAtTenden, S.; Nikiforov; B.; ARMSTRONG, B. G. (2003): «Mortality and temperature in Sofia and London». Journal of Epidemiology and Community Health, 57, 628-633.

PEÑA, J. C.; RASO, J. M. (2008): «Sectorización territorial de los avisos de calor emitidos por el Servei Meteorològic de Catalunya. Aplicación de un análisis en componentes principales a la temperatura máxima diaria». En J. SigRó; M. BRunet; E. Aguilar (Eds.): Cambio climático regional y sus impactos. (pp. 401-412). Tarragona: Asociación Española de Climatología.

Ren, C.; Williams, G. M.; Morawska, L.; Mengersen, K.; Tong, S. (2008): «Ozone modifies associations between temperature and cardiovascular mortality: analysis of the MNMAPS data». Occupational and Environmental Medicine, 65, 255-260. 
Ren, C.; O'NeILl, M. S.; Kyun, P. S.; Sparrow, D.; VoKOnAS, P.; SchWARTZ, J. (2011): "Ambient temperature, air pollution, and heart rate variability in an aging population». American Journal of Epidemiology, 173(9), 1013-1021.

Robine, J. M.; Cheung, S. L.; Le Roy, S.; VAn Oyen, H.; Michel, J. P.; HERRMANN, F. R. (2008): "Death toll exceeded 70.000 in Europe during the summer of 2003». Comptes Rendus Biologies, 331, 171-178.

ROCKLÖV, J.; FORSBERG, B.; MEISTER, K. (2009): «Winter mortality modifies the heat-mortality association the following summer». European Respiratory Journal, 33, 245-251.

RoOney, C.; MCMichael, J.; Kovats, R. S.; Coleman, M. P. (1998): «Excess mortality in England and Wales, and in Greater London, during the 1995 heatwave». Journal of Epidemiology and Community Health, 52, 482-486.

SARTOR, F.; SNACKen, R.; Demunth, C.; WALCKIERS, D. (1995): «Temperature, ambient ozone levels, and mortality during summer, 1994, in Belgium». Environmental Research, 70, 105-113.

SCHÄR, C.; JENDRITZKY, G. (2004): «Hot news from summer 2003». Nature, 432(2), 559-560.

SEmEnZA, J. C. (1996): «Deaths in the Chicago Heat Wave». The New England Journal of Medicine, 335(24), 1848-1849.

SemenzA, J. C.; Mccullough, J. E.; Flanders, W. D.; Mcgeehin, M. A.; LUMPKIN, J. R. (1999): «Excess hospital admissions during the July 1995 heat wave in Chicago». American Journal of Preventive Medicine, 16(4), 269-277.

SemenZA, J. C.; Rubin, C. H.; FAlter, K. H.; SelaniKiO, J. D.; Flanders, W. D.; HowE, H. L. (1996): «Heat-related deaths during the July 1995 heat wave in Chicago». The New England Journal of Medicine, 335(2), 84-90.

SHERIDAN, S. C.; KALKSTEIN, L. S. (2004): "Progress in heat watch-warning system technology”. Bulletin of the American Meteorological Society, 85(12), 19311941.

SMith, K. R.; JeRret, M.; ANDERSON, H. R.; BURnetT, R. T.; StONE, V.; DERWENT, R. Atkinson, R. W.; Cohen, A.; Shonkoff, S. S.; Krewski, D.; Pope, C. A.; Thun, H. J.; Thurston, G. (2009): «Public health benefits of strategies to reduce greenhouse-gas emissions: health implications of short-lived greenhouse pollutants». Lancet 374 (9707), 2091-2103.

Stafoggia, M.; Forastiere, F.; Agostini, D.; Biggeri, A.; BisAnti, L.; CAdum, E.; CARANCI, N.; De'DONATO, F.; De Lisio, S.; DE MARIA, M. (2006): «Vulnerability to heat-related mortality: a multicity, population-based; case-crossover analysis». Epidemiology, 17(3), 315-323.

SteAdman, R. G. (1979): «The assessment of sultriness. Part I: Temperaturehumidity index based in human physiology and clothing science. Part II: Effects of wind, extra radiation and barometric pressure». Journal of Applied Meteorology, 18, 861-885.

SUNYER, J. (2008). «Commentary: evaluating response to heat waves». International Journal of Epidemiology, 37, 317-318.

TAN, J.; KALKSTEIN, L. S.; HUANG, J.; LiN, S.; YiN, H.; SHAO, D. (2004): «An operational heat/health warning system in Shanghai». International Journal of Biometeorology, 48, 157-162. 
TAN, J.; Zheng, Y.; TANG, X.; GuO, C.; LI, L.; Song, G.; Zhen, X.; YUAN, D.; KALKSTEIN, A. J.; LI, F.; CHEN, H. (2010): «The urban heat island and its impact on heat waves and human health in Shanghai». International Journal of Biometeorology, 54; 75-84.

Tobias, A.; García De Olalla, P.; Linares, C.; Bleda, M. J.; CaYlÀ, J. A.; DíAZ, J. (2010): «Short-term effects of extreme hot summer temperatures on total daily mortality in Barcelona, Spain». International Journal of Biometeorology, 54, 115-117.

Van Rossum, C. T. M.; Shirpley, M. J.; Hemingway, H.; GrobBe, D. E.; MACKENBACH, J. P.; MARMOT, M. G. (2001): «Seasonal variation in causespecific mortality: Are there high-risk groups? 25-year follow-up of civil servants from the first Whitehall study». International Journal of Epidemiology, 30, 1109-1116.

VAneckova, P.; Beggs, P. J.; De DeAr, R. J.; Mccracken, K. W. (2008): «Effect of temperature on mortality during the six warmer months in Sidney, Australia, between 1993 and 2004». Environment Research, 108, 361-369.

VigotTi, M. A.; MuggeO, V. M. R. (2006): «The effect of birthplace on heat tolerance and mortality in Milan, Italy, 1980-1989». International Journal of Biometeorology, 50, 335-341.

Whitman, S.; Good, G.; Donoghue, E. R.; Benbow, N.; Shou, W.; Mou, S. (1997): «Mortality in Chicago attributed to the July 1995 heat wave». American Journal of Public Health; 87(9), 1515-1518.

Wilkinson, P.; SMith, K. R.; DAvies, M.; AdAir, H.; AMstrong, B. G.; BARret, M.; Bruce, N.; Haines, A.; Hamilton, I.; Oreszczyn, T.; Ridley, I.; TONne, C.; ChalaBI, Z. (2009): «Public health benefits of strategies to reduce greenhouse-gas emissions: household energy». Lancet, 374(9705), 1917-1929.

Williams, S.; NitschKe, M.; Sullivan, T.; TuCKeR, G. R.; Weinstein, P.; PisAniello, D. L.; PARTOIN, K. A.; BI, P. (2012): «Heat and health in Adelaide, South Australia: Assessment of heat thresholds and temperature relationships». Science of Total Environment, 414, 126-133.

WoOdCOK, J.; EdWARds, P.; AMSTRONG, B. G.; Ashiru, O.; BANister, D.; Beevers, S.; Chalabi, Z.; Chowdhry, Z.; Cohen, A.; Frenco, O.; Haines, A.; HICKMAN, R.; LindSAY, G.; MiTTAL, I.; MOHAN, D.; TIWARI, G.; WOODWARD, A.; ROBERTS, I.. (2009): «Public health benefits of strategies to reduce greenhouse-gas emissions urban land transport». Lancet, 374(9705), 1930-1943.

ZANOBETTI, A.; SCHWARTZ, J. (2008): «Temperature and mortality in nine US cities». Epidemiology, 19(4), 563-570. 